\title{
A Multi-Time-Scale Four-Dimensional Variational Data Assimilation Scheme and Its Application to Simulated Radial Velocity and Reflectivity Data
}

\author{
TAO Sun AND YAODENG CHEN \\ Key Laboratory of Meteorological Disaster of Ministry of Education/Collaborative Innovation Center on \\ Forecast and Evaluation of Meteorological Disasters, Nanjing University of Information Science \\ and Technology, Nanjing, China \\ JUANZHEN SUN \\ National Center for Atmospheric Research, Boulder, Colorado \\ HONGLI WANG \\ Cooperative Institute for Research in the Atmosphere, Colorado State University, Fort Collins, and NOAA/OAR/Earth \\ System Research Laboratory/Global Systems Division, Boulder, Colorado \\ Haiqin Chen, Yuanbing Wang, And Deming Meng \\ Key Laboratory of Meteorological Disaster of Ministry of Education/Collaborative Innovation Center on \\ Forecast and Evaluation of Meteorological Disasters, Nanjing University of Information Science \\ and Technology, Nanjing, China
}

(Manuscript received 19 June 2019, in final form 25 February 2020)

\begin{abstract}
In this study, a multi-time-scale four-dimensional variational data assimilation (MTS-4DVar) scheme is developed and applied to the assimilation of radar observations. The MTS-4DVar employs multitime windows with various time lengths in the framework of incremental 4DVar in the Weather Research and Forecasting Data Assimilation (WRFDA). The objective of MTS-4DVar is to enable the 4DVar data assimilation system to extract multiscale information from radar observations, and the algorithm of MTS4DVar is first discussed in detail. Using a heavy rainfall case, it is shown that the nonlinearity growth of reflectivity is faster than that of radial velocity, suggesting that the time window for assimilating reflectivity in the incremental 4DVar should be shorter than that of radial velocity. A series of single observation tests and observing system simulation experiments (OSSEs) are then presented to examine the physical characteristics and performance of MTS-4DVar. These experiments demonstrate that the MTS-4DVar is capable of combining the larger-scale information from a longer time window and the local-scale features from a shorter time window. With the OSSEs it is shown that the value of the cost function is reduced properly in the minimization of the MTS-4DVar with a combination of longer and shorter time windows. By assimilating the radar radial velocity alone, we found that the MTS-4DVar reduces the analysis and forecast errors and improves the precipitation forecasts in comparison with the normal incremental 4DVar. Additional assimilation of reflectivity further improved the precipitation forecasts, and the results show that the radar reflectivity can also be well assimilated by using MTS-4DVar.
\end{abstract}

\section{Introduction}

Doppler radars can provide high-resolution observations both in space and time, thus they can be used to monitor and study severe weather systems. A variety of data assimilation methods have been developed to

Corresponding author: Yaodeng Chen, keyu@nuist.edu.cn assimilate radar observations to provide numerical weather prediction (NWP) with more accurate initial conditions [see Sun et al. (2014) and Gustafsson et al. (2018) for review].

The three-dimensional variational data assimilation (3DVar) technique has been widely used in radar data assimilation due to its relatively low computational cost and stability (Xiao et al. 2005, 2007; Pu et al. 2009; 
Gao and Stensrud 2012; Wang et al. 2013a; Shen et al. 2017; Liu et al. 2019). But its application on fast-evolving convective systems is limited by its static, homogeneous and isotropic background error covariance (BEC; Gao and Stensrud 2014). The ensemble Kalman filter (EnKF; Evensen 1994) method has been successfully applied to radar assimilation in many studies because of the flow-dependent BEC calculated from ensemble forecasts (e.g., Snyder and Zhang 2003; Tong and Xue 2005; Xue et al. 2006; Dowell et al. 2011). However, the EnKF method suffers rank deficiency and sampling errors from its limited ensemble sizes (Evensen 2004), and it's hard to properly account for the model errors, such as the rapidly growing nonlinear errors, in computing the BEC.

The four-dimensional variational data assimilation (4DVar; Le Dimet and Talagrand 1986; Lewis and Derber 1985) is another advanced technique that has been used for radar data assimilation (Sun and Crook 1997). 4DVar uses a set of prognostic equations as a strong constraint, or weak constraint (Trémolet 2006) to consider model errors, to assimilate observations at multiple times, and its BEC is implicitly flowdependent (Lorenc 2003; Lorenc and Rawlins 2005; Huang et al. 2009). The 4DVar technique has been successfully applied to the global model in the European Centre for Medium-Range Weather Forecasts (ECMWF; Rabier et al. 2000; Mahfouf and Rabier 2000; Klinker et al. 2000) and several 4DVar assimilation systems have been applied to radar assimilation, such as the variational Doppler radar analysis system (VDRAS; Sun et al. 2010), the Japan Meteorological Agency's mesoscale assimilation system (Kawabata et al. 2014; Aranami et al. 2015), the Met Office's 4DVar (Li et al. 2018), and the Weather Research and Forecasting (WRF) 4DVar (Wang et al. 2013b; Sun and Wang 2013).

To reduce the computational cost and improve the mathematical condition of the cost function, the incremental method is commonly adopted in 4DVar (Courtier et al. 1994). The incremental 4DVar uses a sequence of tangent linear models (TLM) as approximations to the nonlinear model as the model constraint, and the minimization process of the cost function uses a strategy with both an outer loop and an inner loop. The minimization of the cost function is carried out in the inner loop, and the nonlinear basic-state trajectory and the first guess are updated in the outer loop (Zhang et al. 2014). The TLM will differ more from the nonlinear model as nonlinearities increase, and the nonlinearities will accelerate along with the time integration in 4DVar (Bonavita et al. 2018). Ways to counteract the nonlinearity in 4DVar are to shorten the length of the time window, to employ more outer loops, or to gradually increase the time windows in the outer loop (Bonavita et al. 2018; Choi et al. 2013).

For radar reflectivity and radial velocity data assimilation, the nonlinearity issue is particularly worth attention. Fabry and Sun (2010) and Fabry (2010) investigated the nonlinearity propagation contributed from radar reflectivity and radial velocity and found that the two had different nonlinearity growth characteristics. Wang et al. (2012) showed that the evolution of cloud condensates is more nonlinear than horizontal wind, temperature, and humidity. These results suggested that the time window for radial velocity should be longer than that for reflectivity. A longer window can assimilate more observations but may decrease the accuracy of the TLM approximation, while a shorter window can have a more accurate TLM approximation but may result in an insufficient time for the information propagation from observed to unobserved variables (Wang et al. 2013a). Therefore, a question arises is how to choose the time window in incremental 4DVar such that it can assimilate as many observations as possible but meanwhile guarantee the validity of the TLM approximation in radar data assimilation.

In this study, we propose a new scheme for effectively assimilating radial velocity and reflectivity in an incremental 4DVar framework. The scheme employs multitime windows with various time lengths in order to extract multiscale information from radial velocity and reflectivity observations, and thus it is named as multitime-scale 4DVar (hereafter MTS-4DVar). The performance of MTS-4DVar is evaluated by a series of observing system simulation experiments (OSSEs) for a heavy rainfall case that occurred in northeastern China.

This article is organized as follows. In section 2, the MTS-4DVar scheme is described. The model configuration and the nonlinearity propagation evaluation for the heavy rainfall case are given in section 3 . Section 4 compares the analysis increments of MTS-4DVar from a single observation experiment with those from normal 4DVar experiments. In section 5, a series of OSSEs are carried out to demonstrate the improved performance of MTS-4DVar for radar assimilation and subsequent short-term prediction. A summary and conclusions are given in section 6 .

\section{Algorithm}

\section{a. The cost function of nonlinear 4DVar}

The analysis step of 4DVar may be seen as minimizing a cost function that measures the misfit between observations and predictions by a nonlinear model along with the time window. The cost function of 4DVar is expressed as follows: 


$$
\begin{aligned}
J\left(\mathbf{x}_{0}\right)= & \frac{1}{2}\left(\mathbf{x}_{0}-\mathbf{x}^{b}\right)^{\mathrm{T}} \mathbf{B}^{-1}\left(\mathbf{x}_{0}-\mathbf{x}^{b}\right)+\frac{1}{2} \sum_{k=1}^{K}\left\{\mathbf{y}_{k}^{o}\right. \\
& \left.-H_{k}\left[M_{k}\left(\mathbf{x}_{0}\right)\right]\right\}^{\mathrm{T}} \mathbf{R}^{-1}\left\{\mathbf{y}_{k}^{o}-H_{k}\left[M_{k}\left(\mathbf{x}_{0}\right)\right]\right\} .
\end{aligned}
$$

The first term on the right in Eq. (1) is the background term, and the second term is the observation term. The vectors $\mathbf{x}_{0}$ and $\mathbf{x}^{b}$ represent the initial atmospheric states and the priori background states, respectively; the observations $\mathbf{y}^{o}$ within the time window are divided into $K$ discrete time slots with each represented by the subscript $k$, and the observation term is the sum in all time slots; $H_{k}$ is the nonlinear observation operator at time $k$, and $M_{k}$ is the nonlinear model operator that propagates the initial atmospheric state to that at time $k$; and $\mathbf{B}$ and $\mathbf{R}$ are the background and observation error covariance matrices, respectively.

The length of the time window and the number of the time slots in 4DVar are usually determined by the available observation types. The length of these time slots is commonly corresponding to the frequency of observations. In each time slot, the observations are regarded as at the same time [i.e., in the middle for most of the time slots except for the first (last) where the observations are at the beginning (end) of the time slot]. For example, if the observations are available every $10 \mathrm{~min}$ and the length of the 4DVar assimilation window is $30 \mathrm{~min}$, the number of the time slots $K$ is 4 , and thus the 4 time slots are from 0 to 5,5 to 15,15 to 25 , and 25 to $30 \mathrm{~min}$, and the observations in each time slot are regarded as at $0,10,20$, and $30 \mathrm{~min}$, respectively.

\section{b. The cost function of incremental 4DVar}

Like most operational data assimilation systems, the WRF 4DVar follows the incremental 4DVar approach (Huang et al. 2009; Zhang et al. 2014). The incremental approach not only reduces the computational cost but also improves the mathematical conditioning of the cost function due to the use of a coarser-resolution TLM and adjoint model (ADM) with simplified representation of physical processes.

In the incremental 4DVar, the analysis increment of the $n$th outer loop is defined as the difference between the initial state of $n$th outer loop and the first guess, which is equal to the analysis state from the previous outer loop:

$$
\delta \mathbf{x}_{0}^{(n)}=\mathbf{x}_{0}^{(n)}-\mathbf{x}_{g}^{(n)}=\mathbf{x}_{0}^{(n)}-\mathbf{x}_{0}^{(n-1)} .
$$

The observation departure from its observed equivalence at time $k$ is defined as the innovation:

$$
\mathbf{d}_{k}^{(n-1)}=\mathbf{y}_{k}^{o}-H_{k}\left\{M_{k}\left[\mathbf{x}_{0}^{(n-1)}\right]\right\} .
$$

The nonlinear operator $H_{k}$ and $M_{k}$ in Eq. (1) can be linearized into $\mathbf{H}_{k}$ and $\mathbf{M}_{k}$ with the linearization assumption:

$$
H_{k}\left\{M_{k}\left[\mathbf{x}_{0}^{(n)}\right]\right\} \approx H_{k}\left\{M_{k}\left[\mathbf{x}_{0}^{(n-1)}\right]\right\}+\mathbf{H}_{k} \mathbf{M}_{k}\left[\mathbf{x}_{0}^{(n-1)}\right] \delta \mathbf{x}_{0}^{(n)} .
$$

Then, the cost function of the full-form 4DVar can be rewritten to that of incremental 4DVar, after some manipulation, as follows:

$$
\begin{aligned}
J\left[\delta \mathbf{x}_{0}^{(n)}\right]= & \frac{1}{2}\left\{\delta \mathbf{x}_{0}^{(n)}-\left[\mathbf{x}^{b}-\mathbf{x}_{0}^{(n-1)}\right]\right\}^{\mathrm{T}} \mathbf{B}^{-1}\left\{\delta \mathbf{x}_{0}^{(n)}\right. \\
& \left.-\left[\mathbf{x}^{b}-\mathbf{x}_{0}^{(n-1)}\right]\right\}+\frac{1}{2} \sum_{k=1}^{K}\left\{\mathbf{H}_{k} \mathbf{M}_{k}\left[\mathbf{x}_{0}^{(n-1)}\right] \delta \mathbf{x}_{0}^{(n)}\right. \\
& \left.-\mathbf{d}_{k}^{(n-1)}\right\}^{\mathrm{T}} \mathbf{R}^{-1}\left\{\mathbf{H}_{k} \mathbf{M}_{k}\left[\mathbf{x}_{0}^{(n-1)}\right] \delta \mathbf{x}_{0}^{(n)}-\mathbf{d}_{k}^{(n-1)}\right\} .
\end{aligned}
$$

\section{c. The cost function of MTS-4DVar}

The MTS-4DVar is designed to assimilate multiscale information from radial velocity and reflectivity observations by using different assimilation window lengths. In the MTS-4DVar algorithm, the interval of the observation time slots in Eqs. (1) and (5) is the same among all outer loops, while the number of the time slots changes with the outer loop. Longer time windows are employed in the first several outer loops to assimilate more observations, and then the window is shortened from the end of the longer time window for the last several outer loops in order to guarantee the TLM approximation for small-scale circulations, such as convective precipitation. With the multitime windows employed in the incremental 4DVar, it is expected that the MTS4DVar can assimilate multiscale information from radar radial velocity and reflectivity. Then the cost function of the MTS-4DVar can be expressed as follows:

$$
\begin{aligned}
J\left[\delta \mathbf{x}_{0}^{(n)}\right]= & \frac{1}{2}\left\{\delta \mathbf{x}_{0}^{(n)}-\left[\mathbf{x}^{b}-\mathbf{x}_{0}^{(n-1)}\right]\right\}^{\mathrm{T}} \mathbf{B}^{-1}\left\{\delta \mathbf{x}_{0}^{(n)}\right. \\
& \left.-\left[\mathbf{x}^{b}-\mathbf{x}_{0}^{(n-1)}\right]\right\}+\frac{1}{2} \sum_{k=1}^{K^{(n)}}\left\{\mathbf{H}_{k} \mathbf{M}_{k}\left[\mathbf{x}_{0}^{(n-1)}\right] \delta \mathbf{x}_{0}^{(n)}\right. \\
& \left.-\mathbf{d}_{k}^{(n-1)}\right\}^{\mathrm{T}} \mathbf{R}^{-1}\left\{\mathbf{H}_{k} \mathbf{M}_{k}\left[\mathbf{x}_{0}^{(n-1)}\right] \delta \mathbf{x}_{0}^{(n)}-\mathbf{d}_{k}^{(n-1)}\right\} .
\end{aligned}
$$

Note that the fixed number of the time slots $K$ in Eq. (5) becomes the changeable $K^{(n)}$ in Eq. (6), indicating that the cost function of MTS-4DVar is changed when the time window length is shortened. It should be noted that, for each outer loop, the length of the integration time of the nonlinear model is the same as that of the TLM and 
ADM to ensure the validity of the TLM and ADM approximation (see Fig. 1). When transitioning from the longer window to the shorter window, the first guess of the shorter window cost function is provided by the optimized solution of the longer window in the previous outer loop, which links the two cost function minimization processes.

An example of MTS-4DVar with two outer loops is illustrated in Fig. 1. A longer window is employed in the first outer loop (top panel), which allows the assimilation of observations of more time slots. In contrast, the shorter window in the second outer loop (bottom panel) assimilates observations of less time slots; however, it can provide an improved tangent linear approximation of the nonlinear trajectory because of the reduced window length, thus allowing a better fit of the analysis to the observations that represent the finescale structure of rapid evolving convection. It is important to note that for each chosen window length multiple outer loops can be executed. Figure 1 only shows one outer loop for each window length for the purpose of easy illustration.

The analysis from the normal incremental 4DVar is the optimal solution over a specified time window; the optimal analysis typically emphasizes the time scales of the atmospheric flow that can be well simulated by the forward model within the given time window. The analysis process of MTS-4DVar is a series of optimal solutions from the longer time window to the shorter time window. When the time window is shortened, both the observations and the nonlinear trajectory in the later segment of the longer window do not constrain the solution over the shorter time window, so the optimality with respect to the shorter time window is emphasized in the later outer loops. Because the optimal solution for the longer window is used as the first guess of the shorter window optimization, the final optimal solution is in effect an aggregation of the two optimizations. It is anticipated that using such a methodology in MTS-4DVar will enable the extraction of the multiscale information contained in the observations.

\section{Model configuration, rainfall case, and nonlinearity evaluation}

\section{a. Model configuration and the rainfall case}

The WRF (Skamarock et al. 2008) Model V3.9.1 is chosen as the numerical model in this study, and the data assimilation system is based on the WRF Data Assimilation (WRFDA) 4DVar V3.9.1. All experiments are conducted on the same experimental domain as

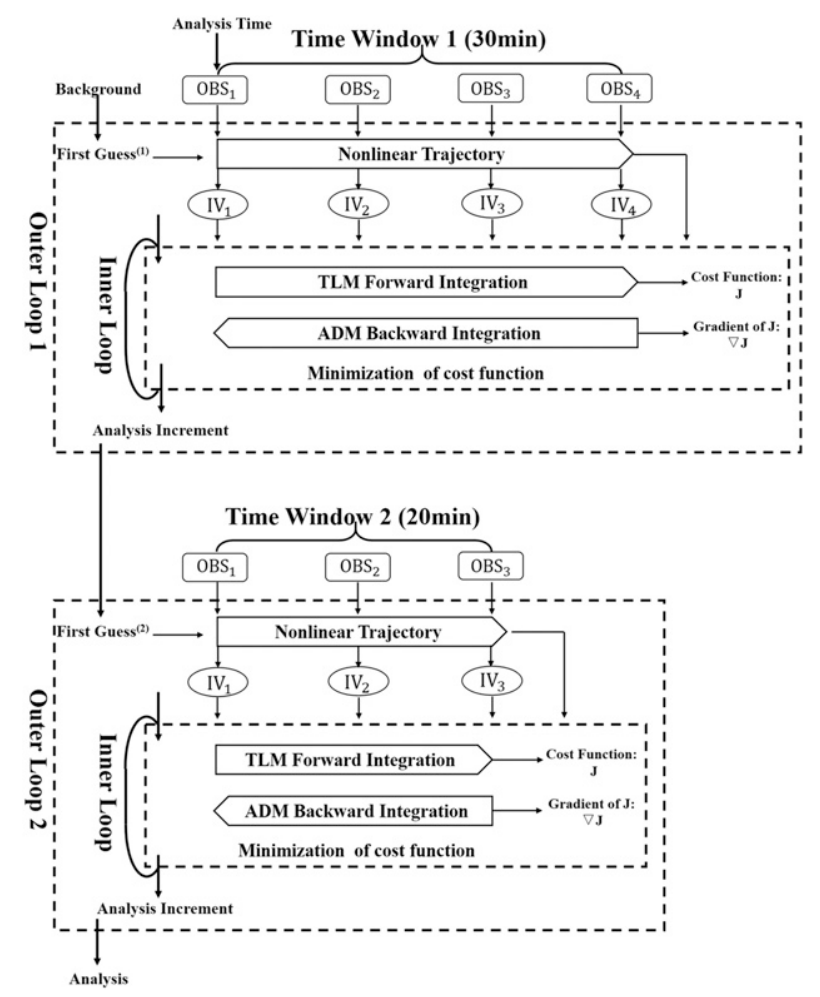

FIG. 1. The flowchart of the MTS-4DVar showing the first outer loop with a 30-min 4D-Var assimilation window (top) assimilating 4 time levels of observations $\left(\mathrm{OBS}_{1}-\mathrm{OBS}_{4}\right)$ and the second outer loop with a reduced 20-min window (bottom) assimilating 3 time levels of observations $\left(\mathrm{OBS}_{1}-\mathrm{OBS}_{3}\right)$. The first guess for the first outer loop is provided by the forecast background while the first guess for the second outer loop is from the longer window analysis. IV stands for innovation.

shown in Fig. 2a. It has two nested domains with the horizontal resolutions of $9 \mathrm{~km}$ (domain 1) and $3 \mathrm{~km}$ (domain 2). The number of horizontal grid points for each domain is $361 \times 301$ and $421 \times 361$, respectively. The number of vertical levels is 41 with the model top at $50 \mathrm{hPa}$, and the pressure profile corresponding to each model level is shown in Fig. 2b. The selected physical parameterizations are WRF double-moment 6-class (WDM6) microphysics scheme (Lim and Hong 2010), the Rapid Radiative Transfer Model (RRTM) longwave radiation scheme (Mlawer et al. 1997) and Dudhia shortwave radiation scheme (Dudhia 1989), the Yonsei University (YSU) planetary boundary layer scheme (Hong et al. 2006), and the Tiedtke cumulus parameterization scheme (Tiedtke 1989). The cumulus scheme is only applied on the coarser grid.

The static BEC is estimated using the National Meteorological Center (NMC) method (Parrish and Derber 1992), which uses the differences between 24- and 12-h forecasts valid at the same time during 

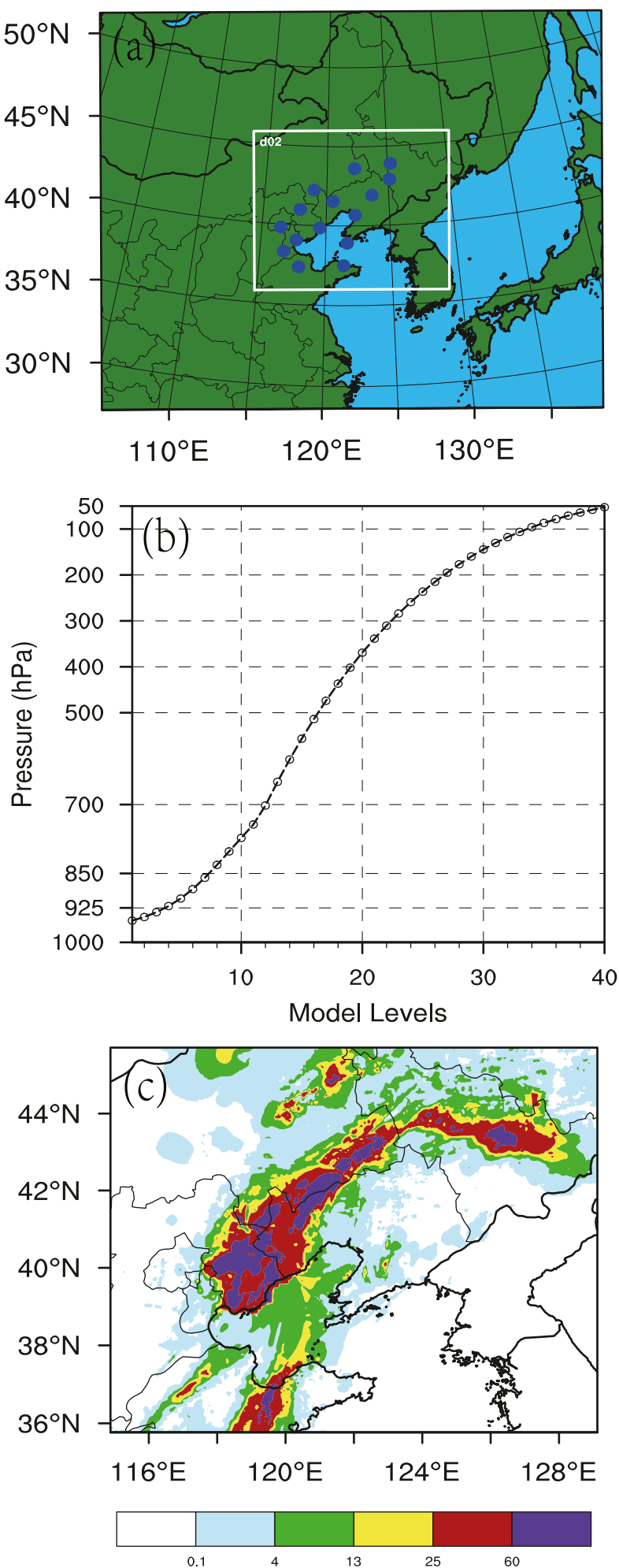

FIG. 2. (a) Experimental domain and radar locations (blue dots), (b) vertical profiles of pressure with respect to model level, and (c) 6-h accumulated precipitation valid at 0000 UTC 3 Aug 2017. a 1-month period (20 July-20 August 2016). The selected control variables in this study are eastward and northward velocity components $(U, V)$, surface pressure $\left(P_{s}\right)$, temperature $(T)$, pseudo-relative humidity $\left(\mathrm{RH}_{s}\right)$, rainwater mixing ratio $\left(Q_{\text {rain }}\right)$, and cloud water mixing ration $\left(Q_{\text {cloud }}\right) ; U$ and $V$ are selected as the momentum control variables in order to better assimilate radar radial velocity observations, and no multivariate operators are considered in the background error covariance because the correlations between the momentum variables and the other variables are insignificant at the convective scale (Sun et al. 2016).

Under the joint influence of the weakened low pressure of typhoon "Haitang" and the subtropical high, from 1200 UTC 2 August to 0000 UTC 5 August 2017, the Liaoning province in northeast China was hit by a heavy rainfall event. Among the 1612 meteorological observation stations in the Liaoning province, $68 \mathrm{ob}-$ served daily accumulated precipitation greater than $250 \mathrm{~mm}$, and 296 observed daily precipitation between 100 and $250 \mathrm{~mm}$, and the maximum hourly accumulated precipitation reached $112.5 \mathrm{~mm}$. In this study, the early stage of the heavy rainfall occurred from 1800 UTC 2 August to 0000 UTC 3 August is investigated. Figure $2 \mathrm{c}$ shows the 6 -h accumulated precipitation from the China Hourly Merged Precipitation Analysis (CHMPA; Shen et al. 2014) valid at 0000 UTC 3 August 2017.

\section{b. Nonlinearity evaluation for the rainfall case}

Since the motivation of the MTS-4DVar scheme is to better assimilate observations with different nonlinear error growth rate during model integration, here we show an example how the model variables $U / V$ and $Q_{\text {vapor }} / Q_{\text {rain }}$, which are directly related to observed radial velocity or reflectivity, present different nonlinearity propagation characteristics. We use the nonlinearity index (NLI) and contradictory information index (CII) (Fabry and Sun 2010) calculated from WRF simulations for such purpose. The NLI measures the magnitude of the departure between the nonlinear and linear states while the CII measures whether the nonlinear and linear states are contradictory with each other, or evolve toward different directions. If the forecast model is linear, a perturbation $\Delta \mathbf{x}$ added to the model state $\mathbf{x}_{0}$ will result in a linear response:

$$
M\left(\mathbf{x}_{0}+\Delta \mathbf{x}\right) \approx M\left(\mathbf{x}_{0}\right)+\Delta \mathbf{x}\left(\frac{\partial M}{\partial \mathbf{x}}\right)_{x_{0}} .
$$

If Eq. (7) stands, a perturbation that is a fraction $l$ of the original one should result in the following: 


$$
M\left(\mathbf{x}_{0}+l \Delta \mathbf{x}\right)-M\left(\mathbf{x}_{0}\right) \approx l\left[M\left(\mathbf{x}_{0}+\Delta \mathbf{x}\right)-M\left(\mathbf{x}_{0}\right)\right] .
$$
$\mathbf{x}$, the NLI at the integration time $T$ in the whole domain $D$ can be defined as

$$
\operatorname{NLI}(v, T, \Delta \mathbf{x})=\frac{\int_{D}\left|\left[v\left(\mathbf{x}_{0}+l \Delta \mathbf{x}\right)-v\left(\mathbf{x}_{0}\right)\right]-l\left[v\left(\mathbf{x}_{0}+\Delta \mathbf{x}\right)-v\left(\mathbf{x}_{0}\right)\right]\right| d D}{\int_{D}\left|v\left(\mathbf{x}_{0}+l \Delta \mathbf{x}\right)-v\left(\mathbf{x}_{0}\right)\right| d D} .
$$

The two terms in the numerator of Eq. (9) can cancel each other when the model is in its linear stage, and NLI is near 0 . If the small perturbation in the initial fields grows significantly with the integration time $T$, the first term will dominate and the NLI will approach 1 . The greater the value of NLI is, the more nonlinear the variable $v$ is. The CII is defined as

$$
\mathrm{CII}(v, T, \Delta \mathbf{x})=\frac{\int_{D}\left|\operatorname{sgn}\left[v\left(\mathbf{x}_{0}+l \Delta \mathbf{x}\right)-v\left(\mathbf{x}_{0}\right)\right]-\operatorname{sgn}\left[v\left(\mathbf{x}_{0}+\Delta \mathbf{x}\right)-v\left(\mathbf{x}_{0}\right)\right]\right| d D}{\int_{D}\left|\operatorname{sgn}\left[v\left(\mathbf{x}_{0}+l \Delta \mathbf{x}\right)-v\left(\mathbf{x}_{0}\right)\right]+\operatorname{sgn}\left[v\left(\mathbf{x}_{0}+\Delta \mathbf{x}\right)-v\left(\mathbf{x}_{0}\right)\right]\right| d D},
$$

where

$$
\operatorname{sgn}(x)=\left\{\begin{array}{r}
1: x>0 \\
0: x=0 \\
-1: x<0
\end{array}\right.
$$

The CII has smaller values when the nonlinear and linear states have the same signs and larger values otherwise, and it can also measure the effect of assimilation. The higher the value of CII is, the less effect of assimilating $v$ it will be. Similar to Fabry and Sun (2010), the thresholds of 0.5 and 0.1 were used for NLI and CII, respectively, to indicate high nonlinearity in this study.

The calculation of the NLI and CII are conducted as follows. First a control run was generated by initializing WRF at 1200 UTC 2 August 2017 with the $0.5^{\circ} \times 0.5^{\circ} \mathrm{GFS}$ analysis. Perturbations of each variable were then added to the 6-h forecast at 1800 UTC 2 August to create two perturbed runs; the perturbations added by the second run were only one-eighth of that of the first run. The 3-h forecasts were then carried out with the perturbed initial conditions, and the results were output every $5 \mathrm{~min}$. 20 pairs of such perturbed runs were generated with different perturbations added at the same time. The outputs of the control run and each pair of the perturbed runs were used to calculate NLI and CII, and the final results were then averaged over the 20 pairs of perturbed runs.

Figure 3 shows the NLI and CII values with respect to the integration time for $U, V, Q_{\text {vapor }}$, and $Q_{\text {rain }}$. The NLIs for variables $U$ and $V$ cross the 0.5 threshold in $70 \mathrm{~min}$ in contrast to 25 and 10 for $Q_{\text {vapor }}$ and $Q_{\text {rain }}$, respectively. Similarly, the times taken for $Q_{\text {vapor }}$ and $Q_{\text {rain }}$ to cross the CII $=0.1$ threshold are much shorter and as short as $5 \mathrm{~min}$ for $Q_{\text {rain }}$. The evaluation of the CII and NLI shows that the nonlinearity propagation of the $U, V$ fields (related to radial velocity) is far slower than that of the microphysics (related to reflectivity) and, in the same time window, assimilating radial velocity is more effective than assimilating reflectivity, indicating that the assimilation time window for radial velocity can be longer than that of reflectivity.

\section{Single observation experiments}

In this section, six single observation experiments, which are three assimilating radial velocity and the other three assimilating reflectivity, respectively, are presented to show how observation information is spread with different time windows employed. Different from the usual 3DVar single observation experiment that involves only a single observation, observations from multiple times at a single position are assimilated in this study. Observations of radial velocity or reflectivity at multiple times, computed relative to an assumed radar location of $\left(41.533^{\circ} \mathrm{N}, 120.517^{\circ} \mathrm{E}\right)$, were placed at $\left(41.594^{\circ} \mathrm{N}, 120.055^{\circ} \mathrm{E}\right)$ on the 10 th model level $(2400 \mathrm{~m})$. The observations are assimilated at $0,10,20$, and $30 \mathrm{~min}$ of the 4DVar window. Both the innovation and the observation error of the radial velocity are assumed $1.0 \mathrm{~m} \mathrm{~s}^{-1}$, and the innovation and observation error of 
a) NLI
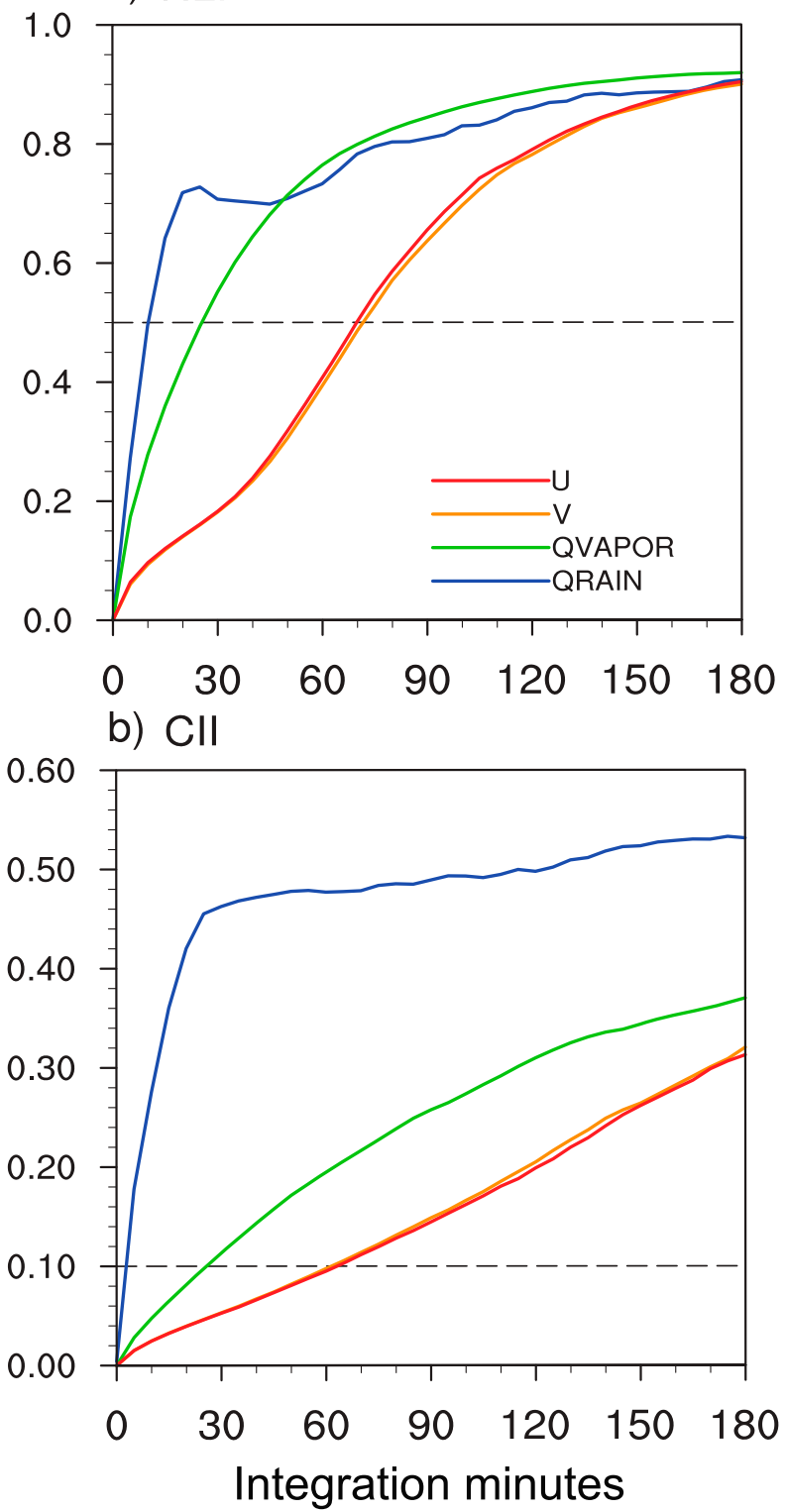

FIG. 3. (a) Nonlinearity index (NLI) and (b) contradictory information index (CII) with respect to time integral (min) for $U, V$, $Q_{\text {vapor }}$, and $Q_{\text {rain }}$.

reflectivity are assumed $3 \mathrm{~dB} Z$ (equivalent to $0.01 \mathrm{~g} \mathrm{~kg}^{-1}$ of rainwater). All the six experiments were conducted with two outer loops. The normal incremental 4DVar experiments S_RV1010 (S_RF1010) and S_RV3030 (S_RF3030) used static lengths of time window in both outer loops, and the lengths were 10 and $30 \mathrm{~min}$, respectively. In the MTS-4DVar experiment S_RV3010 (S_RF3010), the inner loop time windows for the two outer loops were 30 and $10 \mathrm{~min}$, respectively. The details about the time windows of the six single observation experiments are shown in Table 1.
Figure 4 shows the wind, temperature and humidity analysis increments at the 10th model level for S_RV1010, S_RV3030, and S_RV3010 at the start of the assimilation window. For the wind fields, S_RV1010 (Fig. 4a) has the largest increments, but the increments are most localized among the three experiments without showing any dynamical features about $200 \mathrm{~km}$ away from the single observation location. The increments of S_RV3030 (Fig. 4b) are relatively smaller, but spread over a much larger area, indicating that relatively larger-scale features are contained in the analysis increments. The magnitude of the increments for S_RV3010 (Fig. 4c) is between those of the experiments S_RV1010 and S_RV3030, and the shape of the increments for S_RV3010 is also between those two experiments. Unlike the velocity increments which are directly contributed by the radial velocity observation, the increments of temperature and humidity are obtained only through the integrations of the TLM and ADM. For the temperature field, the increments of S_RV1010 (Fig. 4d) are smaller and more localized due to the insufficient time for the model to transfer the observed wind information to temperature with a short time window. S_RV3030 (Fig. 4e) has a greater and broader increment field, but noises appear near the west boundary that may be caused numerically. The temperature increments of S_RV3010 (Fig. 4f) are stronger than S_RV1010 and less noisy than S_RV3030. For the analysis increments of humidity, it is evident that the increments from S_RV3030 have the largest magnitude and areal spread whereas the other two experiments are similar with slightly larger increments for S_RV3010.

The vertical sections of wind speed, temperature and humidity analysis increments along $118.5^{\circ} \mathrm{E}$ are shown in Fig. 5. While the S_RV1010 experiment shows larger wind increments in the low levels around $750 \mathrm{hPa}$ where the observation is located, its temperature and humidity increments are much weaker than those from S_RF3030. On the other hand, although the increments of temperature and humidity from S_RV3030 are obviously larger, the shape and magnitude of its low-level wind increments are noticeably different from those in S_RV1010. In comparison, moderate increments for all three fields are produced by using the varied windows as in S_RV3010.

Figure 6 shows the rainwater increments at the 10th model level responding to the reflectivity assimilation. In S_RF1010, the increments of rainwater are localized and their maximum values are much greater than $0.0025 \mathrm{~g} \mathrm{~kg}^{-1}$. The rainwater increments of S_RF3030 spread a larger area but with smaller values (above $0.002 \mathrm{~g} \mathrm{~kg}^{-1}$ ) when compared to S_RF1010. There were 
TABLE 1. Time window of the single observation experiments.

\begin{tabular}{clc}
\hline \hline Experiments & Time window & Observation \\
\hline S_RV1010 & 10 min for both two outer loops & Radial velocity \\
S_RV3030 & 30 min for both two outer loops 10 min for the second outer loop & Radial velocity \\
S_RV3010 & 30 min for the first outer loop and 10 & Radial velocity \\
S_RF1010 & 10 min for both two outer loops & Reflectivity \\
S_RF3030 & 30 min for both two outer loops & Reflectivity \\
S_RF3010 & 30 min for the first outer loop and 10 min for the second outer loop & Reflectivity \\
\hline
\end{tabular}

negative increments near the single observation site in S_RF3030, and the negative increments are between two maximums of rainwater in the background where the increments are positive. Therefore, the negative increments in S_RF3030 is unreasonable, and it may be caused by the nonlinearity in the longer assimilation window. The increments of S_RF3010 are similar with that of S_RF1010 but with relatively smaller values (about $0.0025 \mathrm{~g} \mathrm{~kg}^{-1}$ ), and the negative increments in S_RF3030 are not found in S_RF3010. Compared with those from the S_RV experiments, the increments for temperature and humidity are rather small (not shown), implying that reflectivity (or hydrometeors) is less coupled with other fields.

The single observation experiments indicate that the longer 4DVar window produces longer-range increments that may represent relatively larger-scale motions, while the shorter window attains strong wind increments close to the observation. A longer time window is necessary to ensure sufficient time to transfer information from observation to unobserved model variables through model dynamics. A longer time window in reflectivity assimilation may bring negative effects likely due to larger nonlinear error growth,
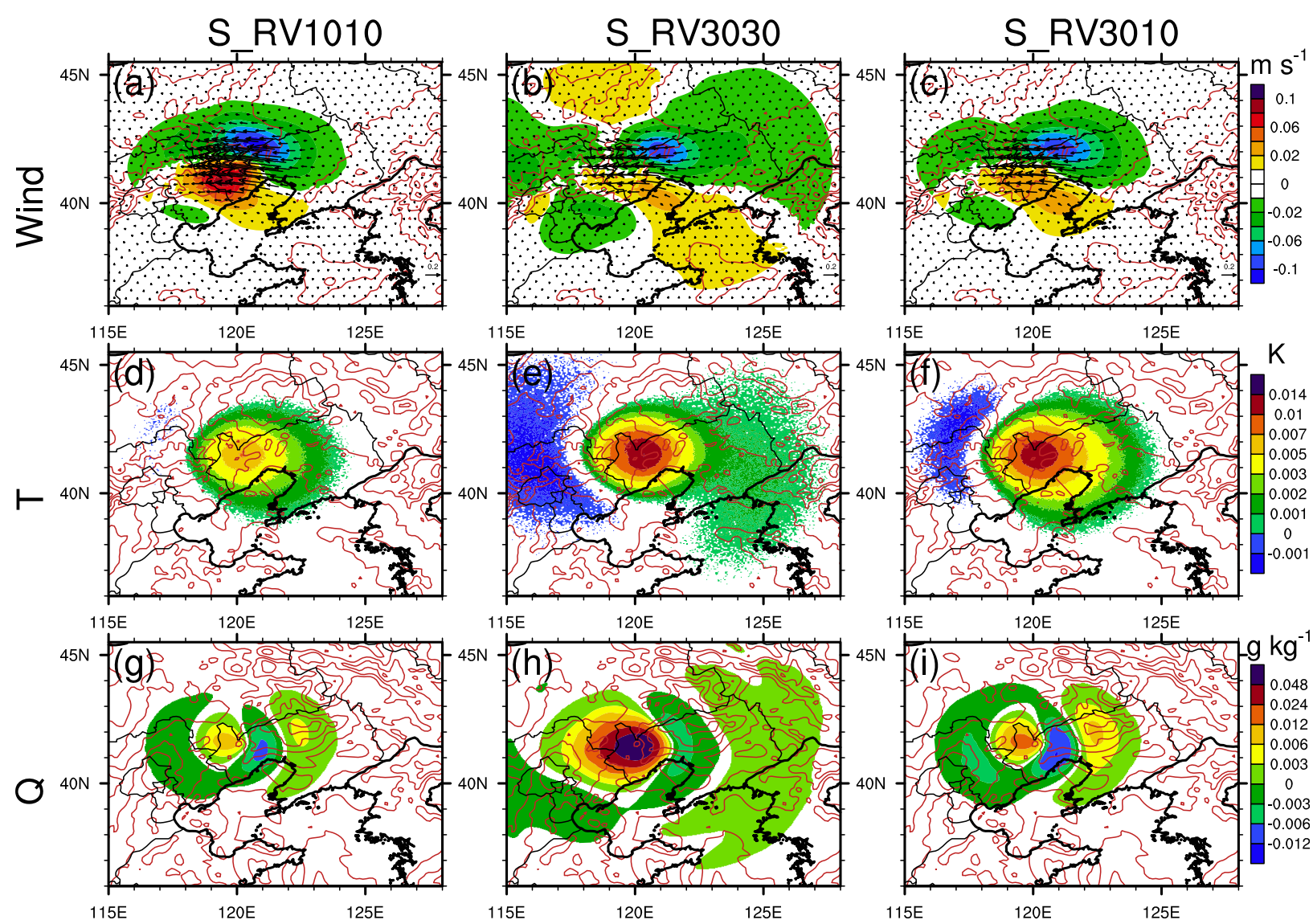

FIG. 4. Wind vectors and speed (vector and shaded, $\mathrm{m} \mathrm{s}^{-1}$ ), temperature (shaded, $\mathrm{K}$ ), and water vapor mixing ratio ( $\mathrm{shaded} \mathrm{g} \mathrm{kg}^{-1}$ ) analysis increments in response to radial velocity assimilation for S_RV1010, S_RV3030, and S_RV3010. 
S_RV1010
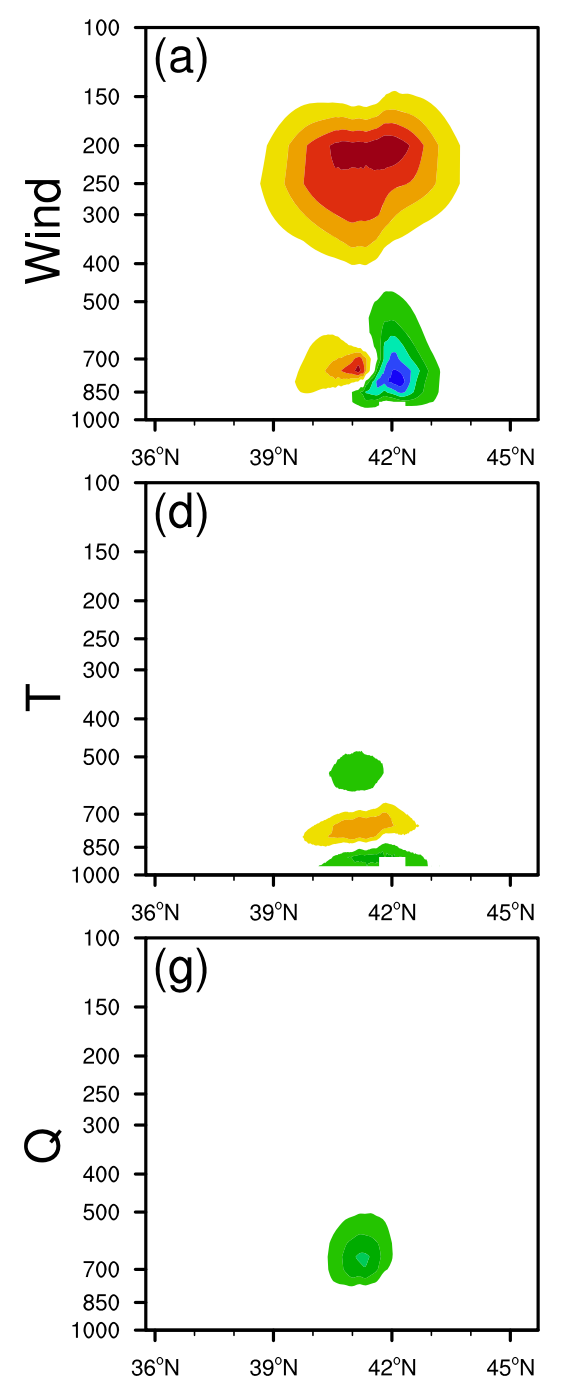

S_RV3030
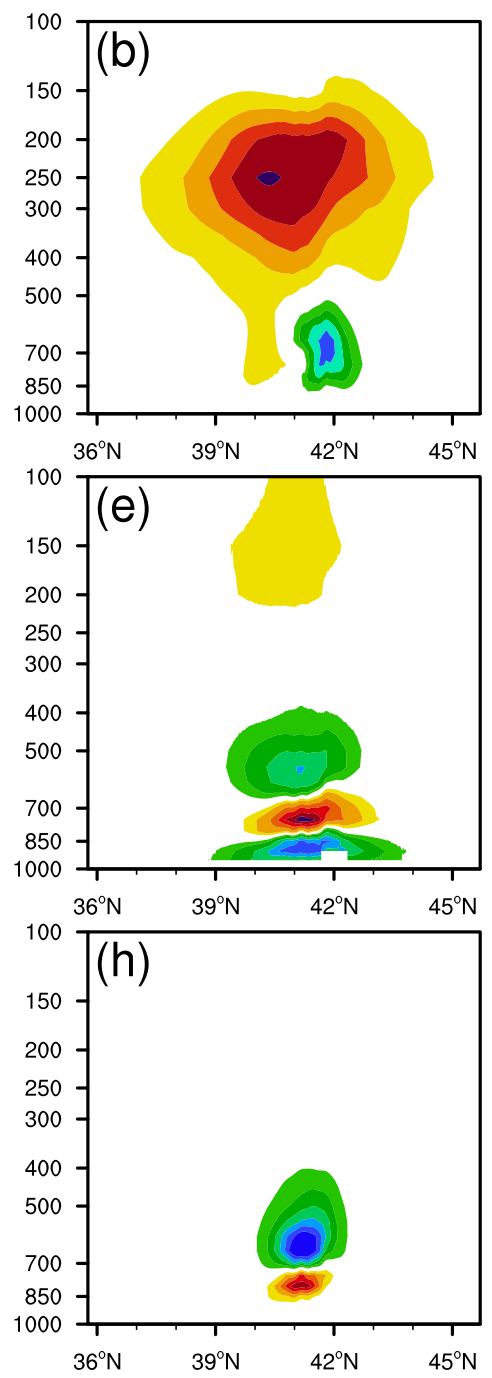

S RV3010
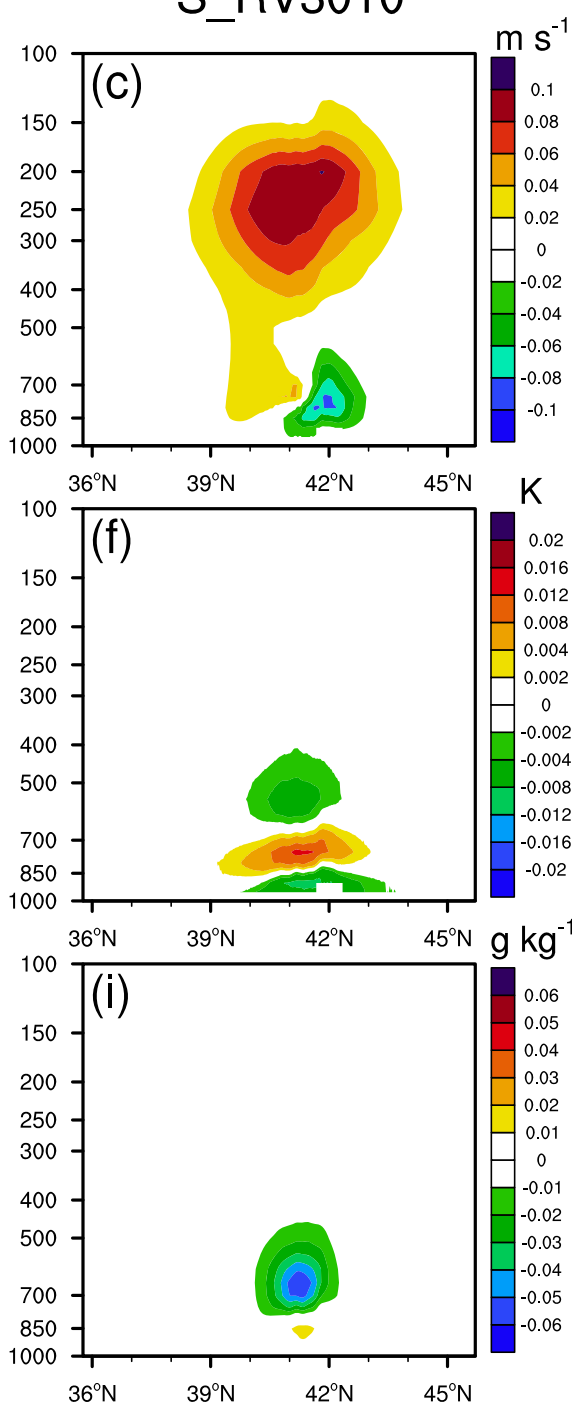

FIG. 5. Vertical sections along $118.5^{\circ} \mathrm{E}$ of analysis increments for wind speed $\left(\mathrm{m} \mathrm{s}^{-1}\right)$, temperature (K), and water vapor mixing ratio $\left(\mathrm{g} \mathrm{kg}^{-1}\right)$ in response to radial velocity assimilation for S_RV1010, S_RV3030, and S_RV3010.

and it can be avoided by using either a shorter time window or multitime windows. In the next section, we will show that the MTS-4DVar can improve analyses by combining multiscale information contained in observations.

\section{Observing system simulation experiments}

In this section, the OSSEs are carried out to investigate the capability of MTS-4DVar in radar radial velocity and reflectivity assimilation.

\section{a. Truth simulation and simulated radar observation}

The truth simulation was generated using a similar procedure described by Zhang et al. (2015). The initial and lateral boundary conditions were provided by the $0.5^{\circ} \times 0.5^{\circ} \mathrm{GFS}$ analysis with random errors added at 0600 UTC 2 August 2017. Then the forecast fields from 1800 UTC 2 August to 0000 UTC 3 August 2017, which have good precipitation forecasts, were selected as the truth simulation. The background fields were provided by the 6 -h forecast, which was initialized at 1200 UTC 2 August using the $0.5^{\circ} \times 0.5^{\circ}$ GFS analysis. The 4DVar data assimilation starts at 1800 UTC 2 August 2017 to initialize simulations that end at 0000 UTC 3 August 2017.

The synthetic radar radial velocity and reflectivity observations were then created from the truth simulation for the 15 radars shown in Fig. 2a. First the observation grid was generated for each radar with 

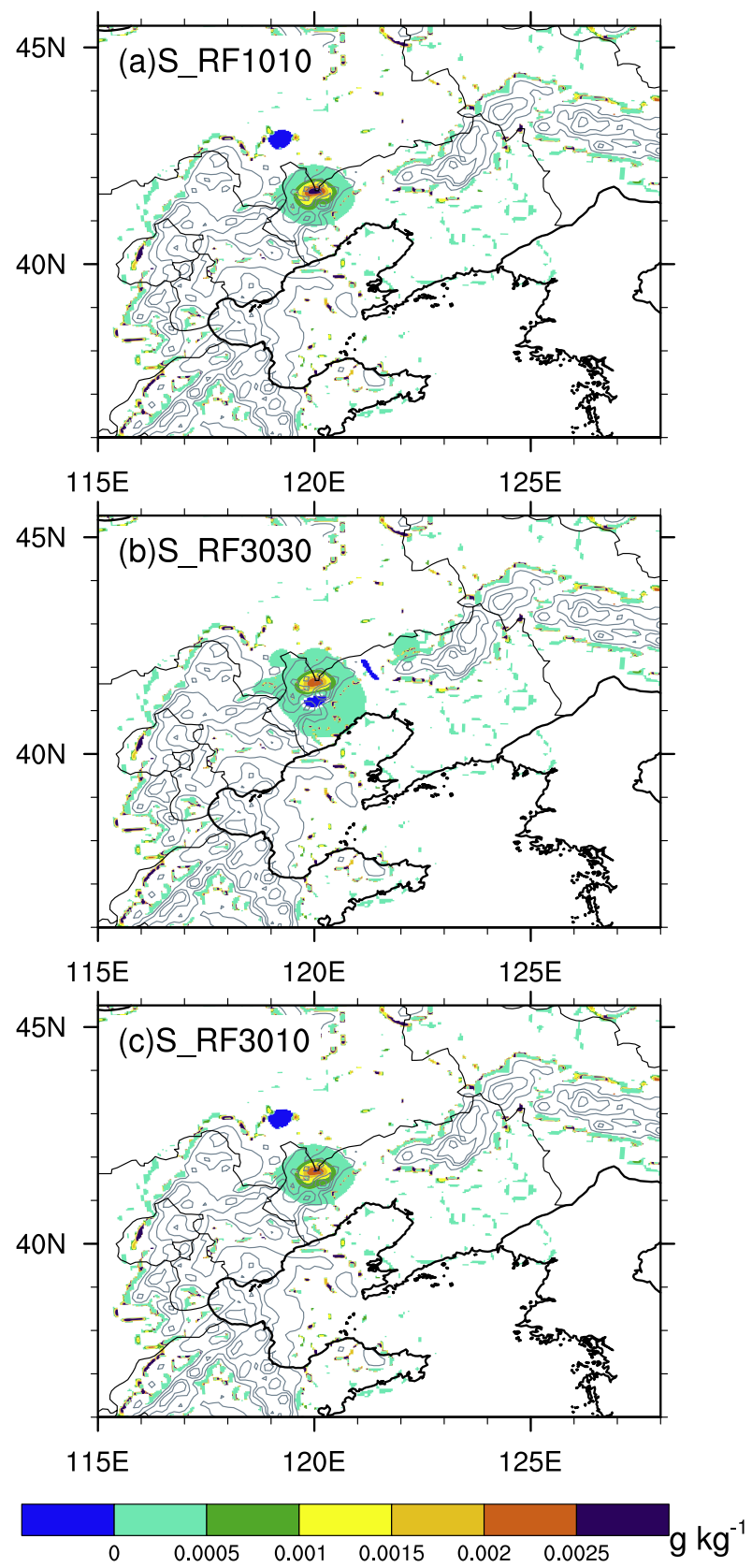

FIG. 6. Rainwater mixing ratio (shaded, $\mathrm{g} \mathrm{kg}^{-1}$ ) analysis increments in response to radar reflectivity assimilation for S_RF1010, S_RF3030, and S_RF3010. The gray lines are the background rainwater mixing ratio.

9 elevations angles $\left(0.5^{\circ}, 1.5^{\circ}, 2.4^{\circ}, 3.4^{\circ}, 4.3^{\circ}, 6.0^{\circ}, 9.9^{\circ}\right.$, $14.6^{\circ}$, and $\left.19.5^{\circ}\right), 3-\mathrm{km}$ range bins up to $240 \mathrm{~km}$, and an azimuthal spacing of $4^{\circ}$. Then radar radial velocity and reflectivity values at model grid points were calculated from the truth simulation using the radar observation operators (Xiao et al. 2005; Gao and Stensrud 2012) and then interpolated to the observation grid points. The Gaussian errors with zero mean and a standard deviation of $1 \mathrm{~m} \mathrm{~s}^{-1}$ and $3 \mathrm{dBZ}$ were added to the synthetic radial velocity and reflectivity, respectively. Considering that errors were introduced in interpolation, the observation errors of radial velocity and reflectivity were inflated to $1.5 \mathrm{~m} \mathrm{~s}^{-1}$ and $5 \mathrm{~dB} Z$.

\section{b. Experimental design}

To evaluate the performance of MTS-4DVar on assimilating radial velocity and reflectivity observations, a series of experiments were conducted as summarized in Table 2. The control experiment, CNTL, was initialized from the background fields whereas the other five experiments assimilated either radar radial velocity only (V1212, V3030, and V3012) or both radial velocity and reflectivity (VF3012 and VF3012A). For all the 4DVar assimilation experiments, two outer loops were employed and 30 iterations were carried out for each outer loop. The length of the time window for the first (second) outer loop was indicated by the first (second) two digits following $\mathrm{V}$ for radial velocity or VF for radial velocity and reflectivity. VF3012A differs from VF3012 in that only $12 \mathrm{~min}$ reflectivity observations were used in VF3012A; that is, the observations from 13 to $30 \mathrm{~min}$ were withheld to avoid the nonlinearity of reflectivity, which was discussed in section $3 \mathrm{~b}$. The assimilation of reflectivity used an indirect scheme that assimilated retrieved rainwater and water vapor estimated from radar reflectivity (Wang et al. 2013a). The latter simply assumes water vapor saturation of $100 \%, 95 \%$, and $85 \%$ for reflectivity above $50 \mathrm{~dB} Z$, within $40-50 \mathrm{dBZ}$, and within $25-40 \mathrm{~dB} Z$, respectively. In this study, the spatial resolution of the TLM and ADM is the same as that of the nonlinear model.

\section{c. Assimilating radial velocity alone}

\section{1) Minimization of THE COST Function AND INITIAL STATE BALANCE}

The cost function and its gradient are discussed to examine whether the MTS-4DVar has a stable behavior in the cost function minimization. The reduction on the cost function and its gradient are shown in Figs. 7a and $7 \mathrm{~b}$, respectively. with respect to the 62 iterations (30 inner loop iterations as well as the initial one for each of the 2 outer loops). In the first outer loop, it is obviously that the shorter time window experiment V1212 has good convergence with a steady reduction on both the cost function and its gradient. A steady reduction on the cost function can be seen in both V3012 and V3030, but quite large gradients are shown during the first several iterations. The second outer loop witnesses a 
TABLE 2. Summary of the six experiments.

\begin{tabular}{llll}
\hline \hline Experiments & \multicolumn{1}{c}{ Time windows } & Observation \\
\hline CNTL & \multicolumn{1}{c}{ No radar observations are assimilated } \\
V1212 & 12 min for both two outer loops & Radial velocity \\
V3030 & 30 min for both two outer loops & Radial velocity \\
V3012 & 30 min for the first outer loop and $12 \mathrm{~min}$ for the second outer loop & Radial velocity \\
VF3012 & 30 min for the first outer loop and $12 \mathrm{~min}$ for the second outer loop & Radial velocity and reflectivity \\
VF3012A & 30 min for the first outer loop and 12 min for the second outer loop & Radial velocity and 12 min reflectivity \\
\hline
\end{tabular}

slight jump in cost functions for V1212 and V3030 because of the updates of the $\mathbf{d}_{k}$ respect to $\mathbf{H}_{k}, \mathbf{M}_{k}$, and of the first guess $\mathbf{x}_{o}$ in Eq. (3). V3012 experiences a sharp reduction on cost function in the second outer loop due to a reduction on the number of observations associated with the shorter time window. Both V1212 and V3012 have good convergence in the second outer loop, but the cost function of V3030 is nearly flat for the longer time window employed. The results indicate that the MTS-4DVar minimization behaves properly and
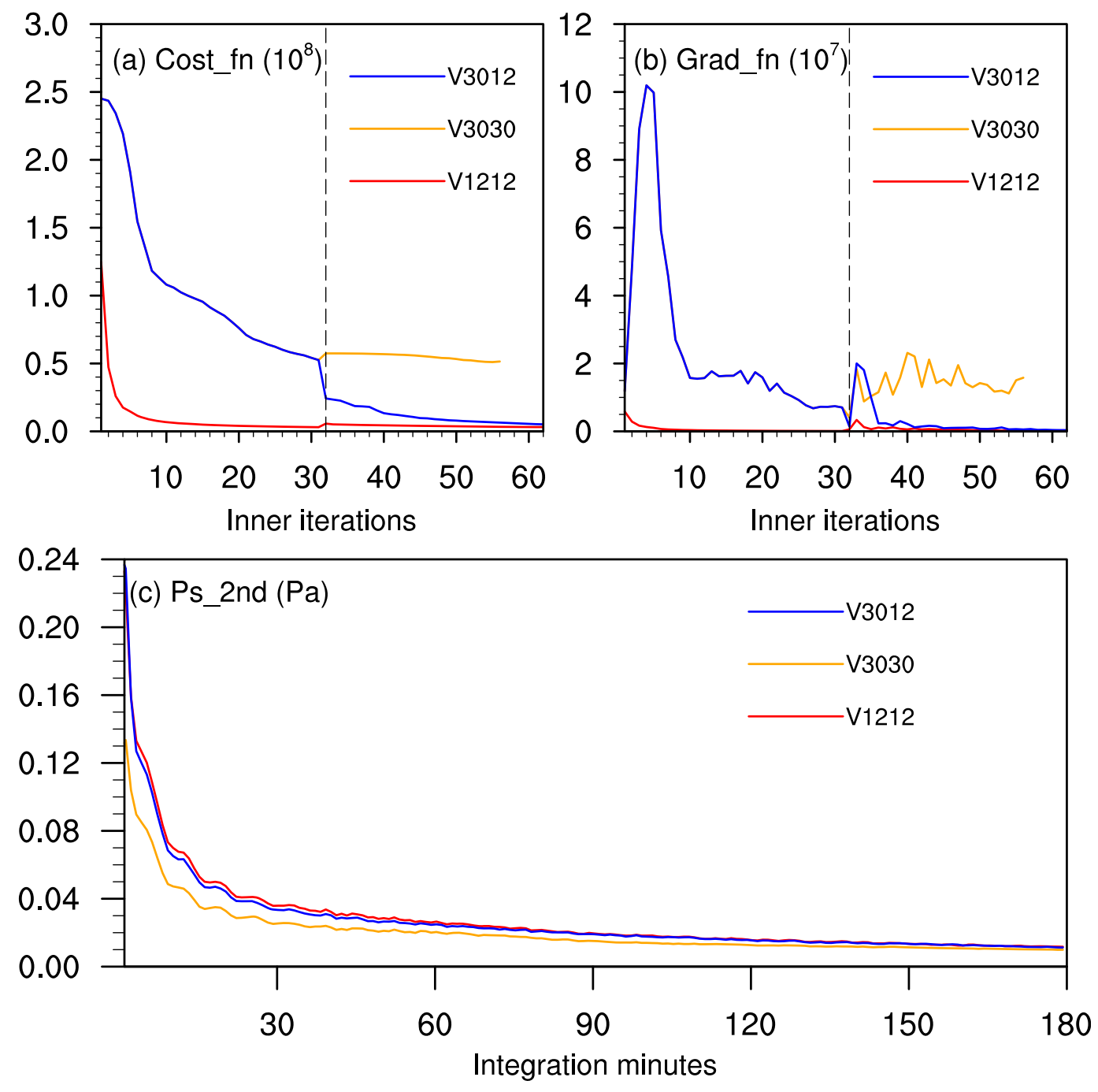

FIG. 7. (a) The cost function, (b) the gradient of cost function with respect to the number of iterations accumulated by two outer loops, and (c) the 6-h evolutions of the mean absolute second time derivative of surface pressure for V1212, V3030, and V3012. In (a) and (b), the first 31 iterations (30 inner iterations and the initial one) to the left of the dotted line are in the first outer loop, while the rest 31 iterations in the second outer loop. 
a) $\mathrm{OmA}$

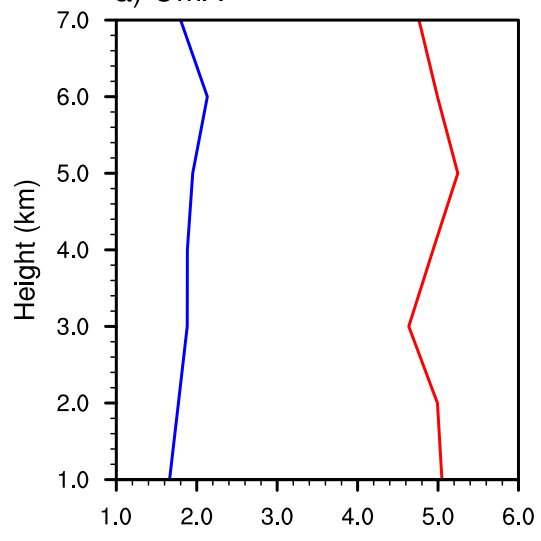

b) $A m B$

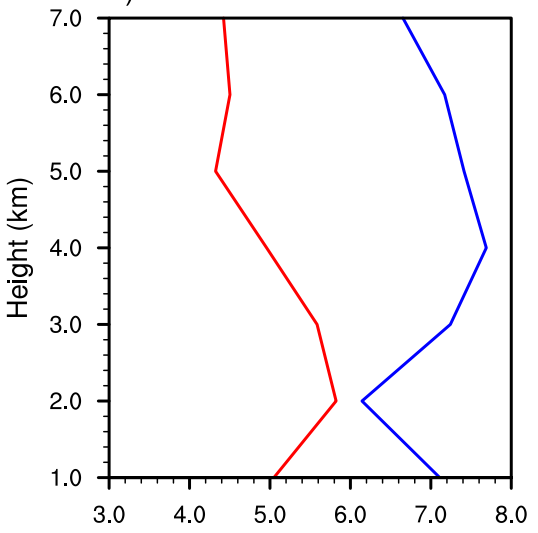

c) $\mathrm{OmF}$

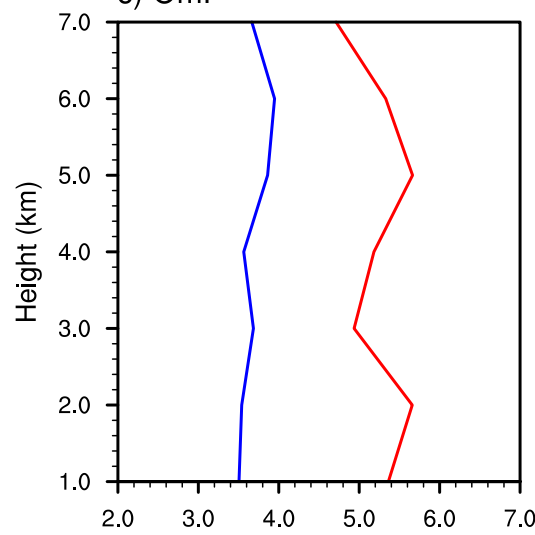

FIG. 8. Vertical profiles of RMSE of the (a) OmA and (b) AmB over the first 12-min window and (c) OmF over the rest 18-min window for radial velocity from the first and second outer loop in V3012.

avoids the unstable minimization from the longer time window in the second outer loop.

To evaluate the initial imbalance characteristics in short-term forecasts based on the analysis from V1212, V3030, and V3012, the mean absolute second derivative of surface pressure (Houtekamer and Mitchell 2005) was calculated. The mean absolute second derivative of surface pressure is defined as

Ps_2nd $=\frac{1}{N_{x} \times N_{y}} \sum_{1}^{N_{y}} \sum_{1}^{N_{x}}\left|p_{s}(t+\Delta t)-2 p_{s}(t)+p_{s}(t-\Delta t)\right|$,

where $p_{s}$ is the surface pressure (hPa); $N_{x}$ and $N_{y}$ are the numbers of horizontal grids, and they are 420 and 360 , respectively; $\Delta t$ is the time step in model integration, and it is $15 \mathrm{~s}$. Ps_2nd reflects the overall balance of the model state. The balance of forecasts measured by Ps_2nd starting form 1800 UTC in the 3-h forecasts is presented in Fig. 7c. In the first hour, the second derivative of surface pressure of the three experiments is reduced from more than 0.12 to about $0.025 \mathrm{hPa}$, and the values are stabilized afterward. V1212 is the most imbalanced among the three experiments, which is not surprising because it misses certain larger-scale and multivariate information by using a smaller window. The use of a longer time window gives the 4DVar sufficient time to propagate the radial wind information to the other variables, thus V3030 is the least unbalanced. By using both the longer and shorter windows the imbalance in V3012 is slightly reduced from that of V1212 in the first $60 \mathrm{~min}$.
Since in the MTS-4DVar experiment V3012 the observations in the later time slots in the first outer loop no longer constrain the minimization in the second outer loop, a question arises whether that will cause the solution of the cost function moving back to the background. To answer that question, we compare the Observations minus Analysis (OmA) and Analysis minus Background $(\mathrm{AmB})$ over the first 12-min window and Observations minus Forecasts $(\mathrm{OmF})$ over the rest 18-min window for radial velocity from the second outer loop with their respective counterparts from the first outer loop. In Fig. 8, the vertical distributions of the root-mean-square errors (RMSEs) of AmB, OmA, and OmF from the two outer loops are compared. The comparison clearly shows that OmA and OmF from the second outer loop is smaller than that from the first outer loop and vice versa for AmB. Apparently, the second outer loop increases the RMSE of AmB and decreases that of OmA, meaning that the minimization solution of the MTS-4DVar experiment in the second outer loop has moved away from the background but toward the observations over the shorter time window. The RMSE of OmF over the later 18-min window is also decreased in the second outer loop, meaning that the improved analysis over the shorter time window can also lead to an improvement of the forecast trajectory over the longer window.

\section{2) ANALysis}

We first examine the differences in the analysis increments among the experiments and the results from the forecasts will be compared in section 5c(3). Figure 9 


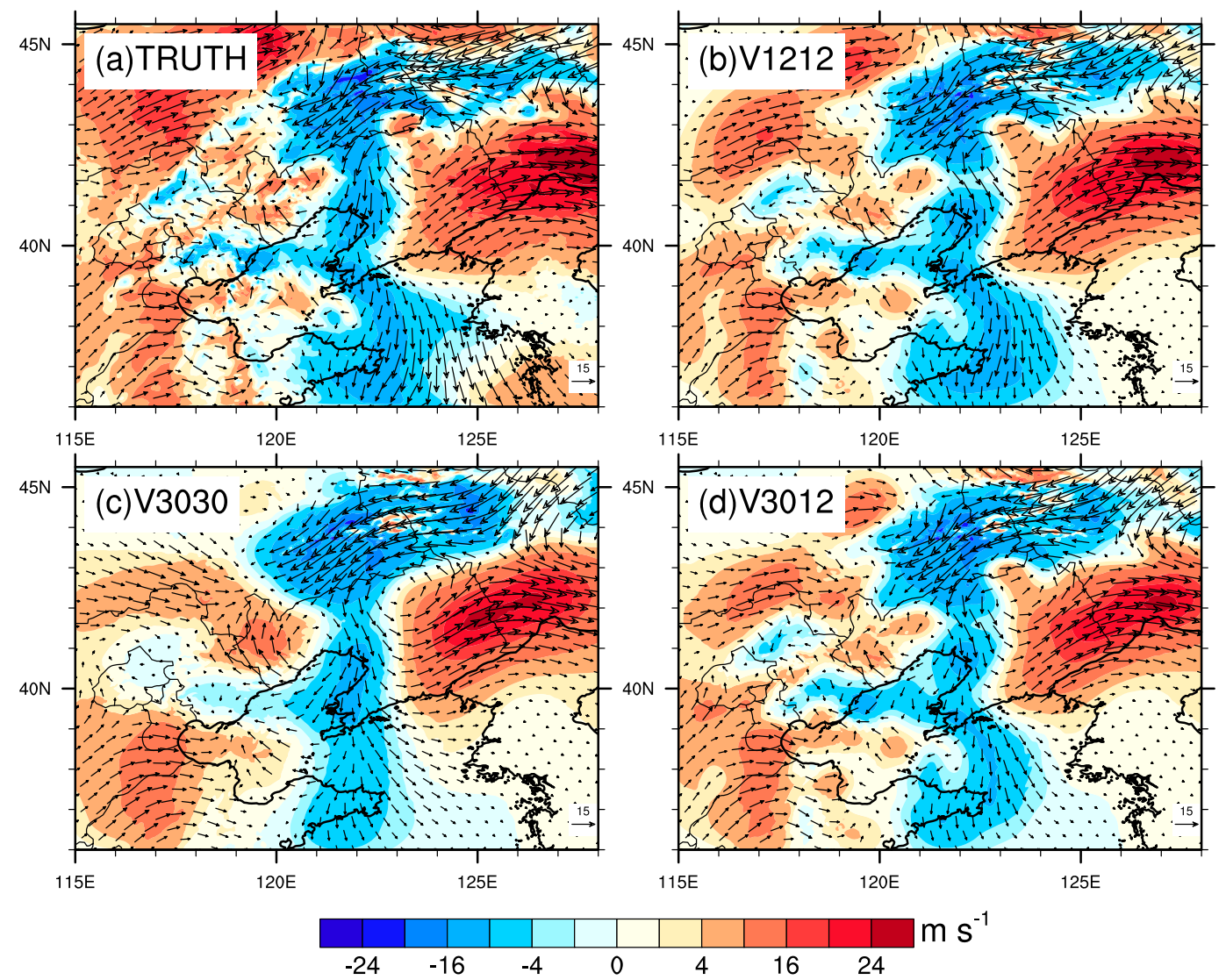

FIG. 9. The $U, V$ winds (vectors; $\mathrm{m} \mathrm{s}^{-1}$ ) and speed (shaded; $\mathrm{m} \mathrm{s}^{-1}$ ) at the 15 th model level for (a) TRUTH minus background, and analysis increments of (b) V1212, (c) V3030, and (d) V3012.

shows the horizontal increments of wind fields at model level 15 (about $500 \mathrm{hPa}$ ). We first notice that the increments of V3030 (Fig. 9c) are smoother and weaker, missing many small-scale features present in the truth run (Fig. 9a, between $117^{\circ}$ and $121^{\circ} \mathrm{E}$ ) that are caused by the convective system (Fig. 2c), although the large-scale pattern (convective environment) is adequately captured. In comparison, the increments of V1212 (Fig. 9b) and V3012 (Fig. 9d) not only capture the large-scale pattern as in Fig. 9c but also add most of the small-scale details associated with the convection. As will be seen next, the main differences between V3012 and V1212 are further revealed in the temperature and water vapor analyses.

The vertical cross sections of the temperature and water vapor increments along $118.5^{\circ} \mathrm{E}$ are shown in Fig. 10. For the temperature fields, the main pattern of the negative differences in the lower levels and positive differences in the middle and upper levels between the truth simulation and the first guess (Fig. 10a) is captured by all the three assimilation experiments. V1212 misses the negative region on the middle levels near $39.5^{\circ} \mathrm{N}$, while both V3012 and V3030 successfully capture it. For the water vapor fields, the increments of V1212 are almost all negative, while V3012 and V3030 successfully analyze the positive patterns in lower and middle levels near $37.5^{\circ} \mathrm{N}$.

The analysis increments shown above suggest that the MTS-4DVar is able to capture the larger-scale and localized dynamical information with a combination of longer and shorter time windows. The results also indicate that the shorter window data assimilation ensuing the longer window in MTS_4DVar does not cause any degradation of the temperature and humidity analyses of those obtained from the longer window alone (V3030); or in other words the information gained from the longer window assimilation is well maintained during the successive shorter window assimilations. Next, we will compare these experiments with quantitative verification against the truth simulation to further evaluate the benefit of MTS-4DVar.

Figure 11 displays the vertical profiles of RMSE between the analysis and the truth simulation. The analysis errors are obviously reduced after the 4DVar 


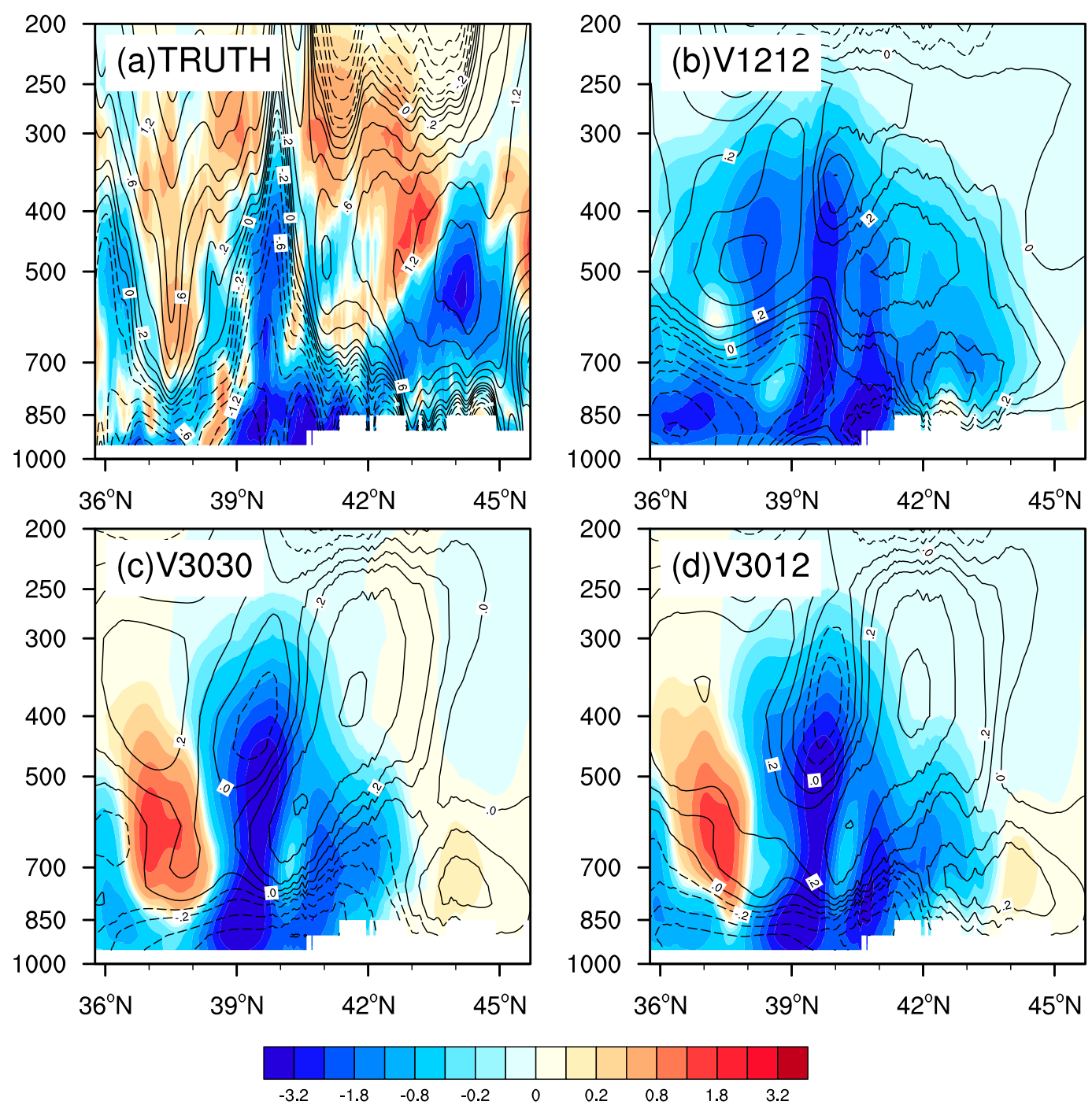

FIG. 10. The vertical sections of temperature (line; K) and water vapor mixing ratio (shaded; $\mathrm{g} \mathrm{kg}^{-1}$ ) along $118.5^{\circ} \mathrm{E}$ for (a) TRUTH minus background, and analysis increments of (b) V1212, (c) V3030, and (d) V3012.

assimilation of radial velocity. For variables $U$ and $V$, the experiment V1212 has the least errors and V3030 has the largest errors among the three assimilation experiments, and the errors of the experiment V3012 are between those two. For variable $T$, the experiment V3012 has the least errors below $500 \mathrm{hPa}$ except for the levels near $700 \mathrm{hPa}$, while V1212 performs the worst below $850 \mathrm{hPa}$. For the humidity variable $Q$, the result of V3012 is similar to V3030, and V1212 even performs worse than CNTL. The better results produced by the experiments V3030 and V3012 are attributed to the use of the $30 \mathrm{~min}$ window, which enables the wind increments to be transferred to those of temperature and humidity through the longer forward model integration.

\section{3) FORECAST}

The quantitative precipitation forecast (QPF) is a vital indicator for evaluating the performance of assimilation and forecasting. Figure 12 presents the 6-h accumulative precipitation initialized from 1800 UTC 2 August for TRUTH, CNTL, V1212, V3030, and V3012. Two main precipitation maxima are simulated by the truth simulation, which are represented by areas A and B in Fig. 12a. The CNTL (Fig. 12b) fails to simulate the precipitation in area $\mathrm{B}$ and overestimated the intensity in area A, but the situation is greatly improved after the assimilation of radial velocity using 4DVar. V1212 (Fig. 12c) slightly improves the precipitation in area $\mathrm{B}$, but it significantly underestimates precipitation in area A. V3030 (Fig. 12d) overestimates the precipitation in area A, but 

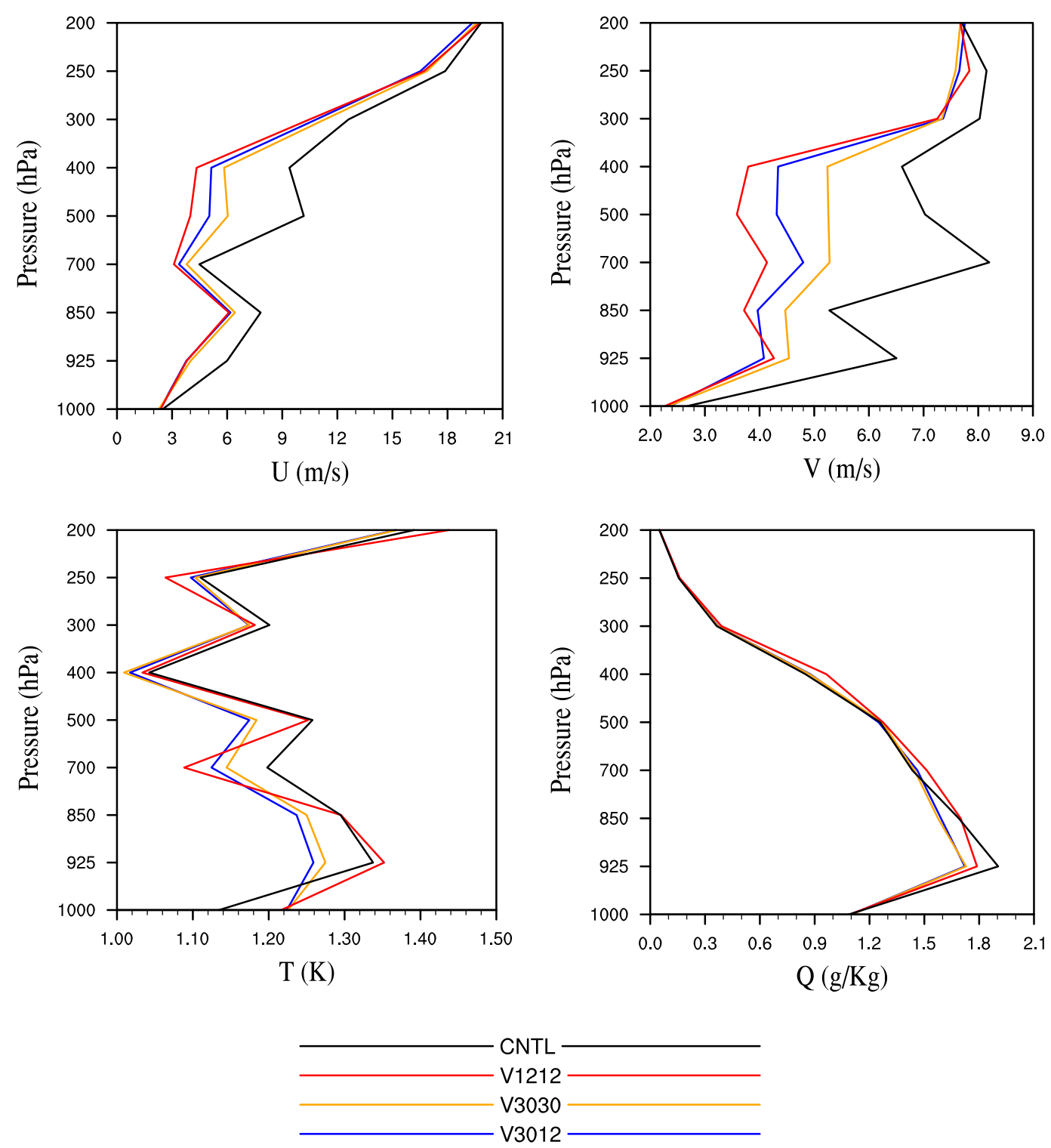

FIG. 11. Vertical profiles of RMSE of the analysis against the truth simulation for (a) $U$, (b) $V$, (c) $T$, and (d) $Q$ of CNTL, V1212, V3030, and V3012.

the precipitation in area B is largely underestimated; V3012 (Fig. 12e) also underestimates the precipitation in area $\mathrm{B}$, but it has the best precipitation forecasts in area $\mathrm{A}$.

To quantitatively evaluate the precipitation forecasts of each experiments, the fractions skill score (FSS, Roberts and Lean 2008) with the radius of influence of $6 \mathrm{~km}$, are computed. The results of FSSs of $6-\mathrm{h} \mathrm{ac}-$ cumulated precipitation valid at 0000 UTC 3 August for CNTL, V1212, V3030, and V3012 with different thresholds are shown in Fig. 13. The score of V3012 is the highest, and that of CNTL is the lowest. V3030 outperforms V1212 in the thresholds between 25 and $60 \mathrm{~mm}$ while V1212 does better in the other three sets of thresholds. Figure 14 displays the hourly accumulated precipitation FSSs with respect to 6-h forecast time with different sets of thresholds. In thresholds between 0.1 and $1.5 \mathrm{~mm}$, the results of FSSs are mixed, but the FSSs of V1212 are slightly higher than those of the other three experiments. The FSSs of V3012 are the largest in thresholds between 1.5 and 7, 7 and 15, and 15 and $40 \mathrm{~mm}$ at almost all forecast hours. V1212 outperforms V3030 at the first two or three forecast hours, but they are reversed afterward. The results of the precipitation forecasts indicate that the MTS-4DVar can effectively improve the 0-6-h QPF using two different window lengths for radial velocity assimilation. 


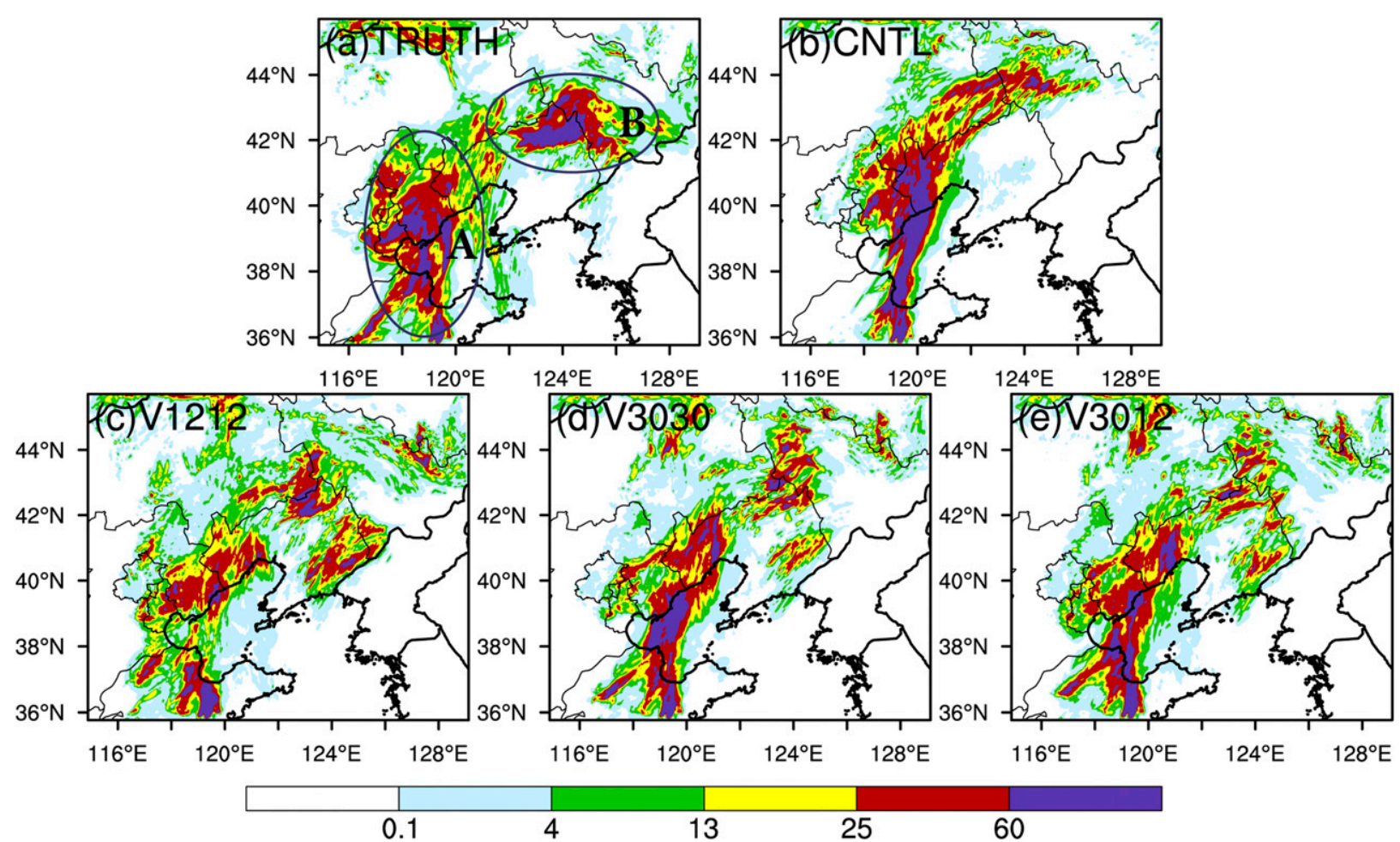

FIG. 12. The 6-h accumulated precipitation (mm) initialized from 1800 UTC 2 Aug 2017 for (a) TRUTH, (b) CNTL, (c) V1212, (d) V3030, and (e)V3012.

The vertical profiles of RMSEs for 6-h forecasts against truth simulation are shown in Fig. 15. V3012 has the least RMSE values for wind variables $U$ and $V$ above $700 \mathrm{hPa}$, and even V3030 outperforms V1212 in wind fields. For temperature, the errors of V3012 and V3030 are similar in the levels between 400 and $850 \mathrm{hPa}$, and V1212 has the largest errors above $850 \mathrm{hPa}$ while V3030 has the largest errors below $850 \mathrm{hPa}$. The forecast errors for $Q$ of V3012 and V3030 are similar, and they are all less than that of V1212. Even though V1212 has least analysis errors in wind fields, the superiorities decrease quickly as the forecast time increases. It may be explained by that the imbalance between analysis variables in V1212 brings negative impacts to forecasts. Benefiting from the larger-scale information from the longer time window and localized information from the shorter window in wind fields and balanced analysis among the observed and unobserved variables, V3012 has the least RMSEs of the 6-h forecasts almost for all variables among the three experiments, and it may explain why the precipitation forecast is well improved by V3012.

\section{d. Assimilating both radial velocity and reflectivity}

Previous studies have shown that the radar observations will be efficiently utilized when radial velocity and reflectivity are assimilated simultaneously (Tong and Xue 2005; Kawabata et al. 2011; Gao and Stensrud 2014). In this subsection, we will focus on the application of MTS-4DVar for the assimilation of both radial velocity and reflectivity. Since it was shown that the V3012 had certain superiority over V1212 and V3030, we added two experiments to assimilate radial

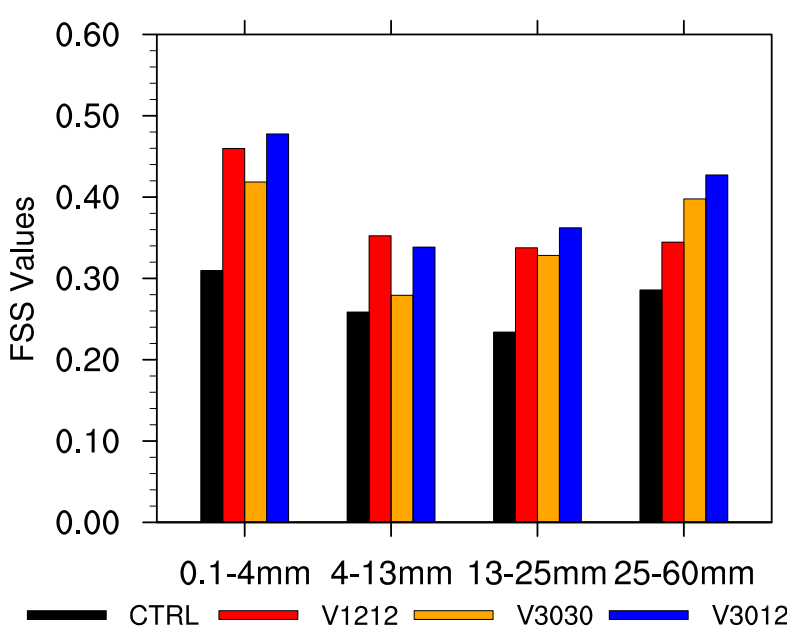

FIG. 13. The FSSs of 6-h accumulated precipitation with different thresholds for CNTL, V1212, V3030, and V3012. 
a) $0.1-1.5 \mathrm{~mm}$

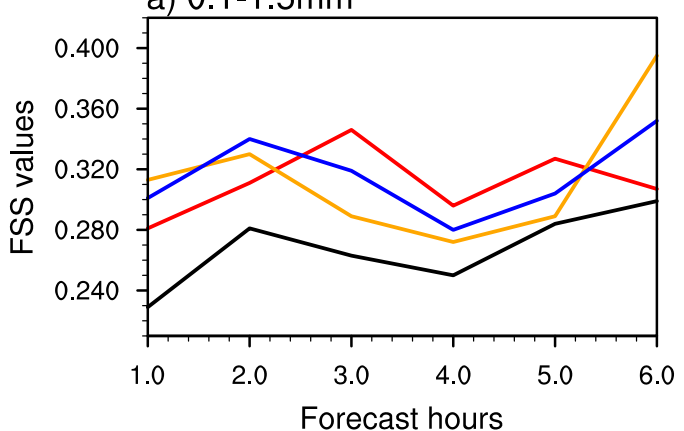

c) $7-15 \mathrm{~mm}$

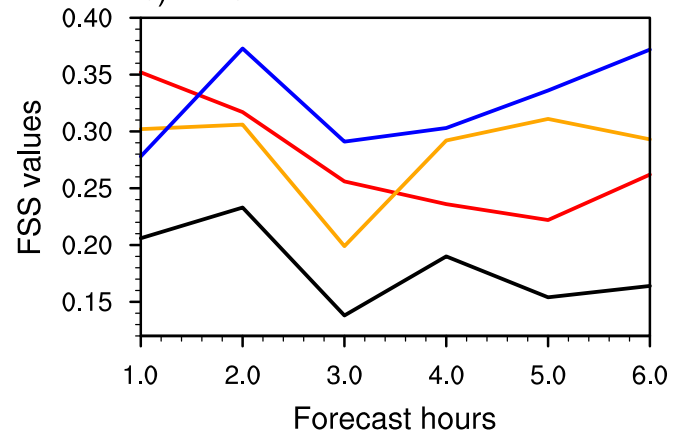

b) $1.5-7 \mathrm{~mm}$

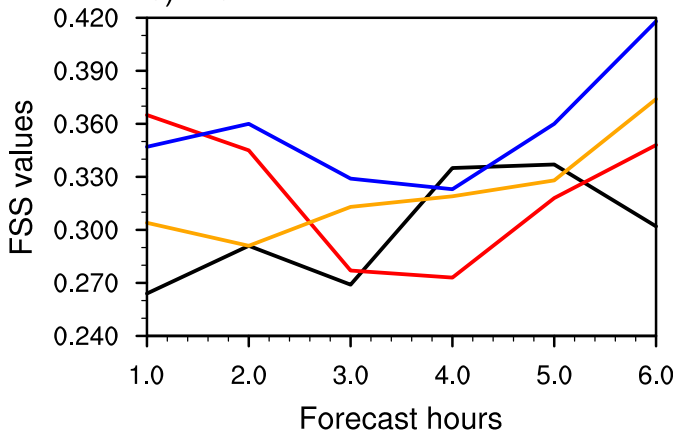

d) $15-40 \mathrm{~mm}$

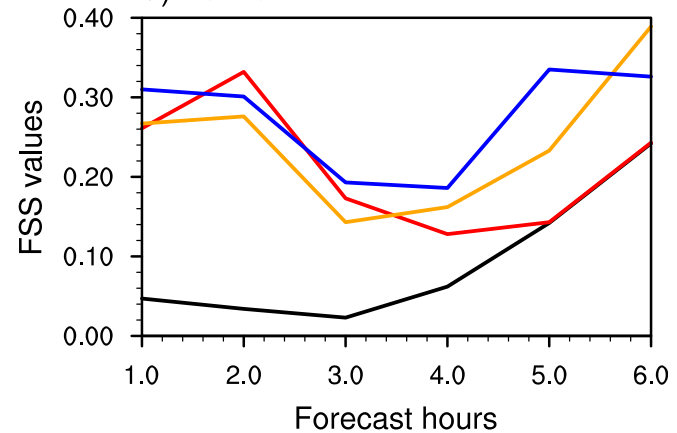

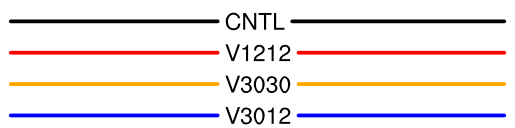

FIG. 14. The FSSs of hourly precipitation with thresholds of (a) $0.1-1.5$, (b) $1.5-7$, (c) 7-15, and (d) $15-40 \mathrm{~mm}$ for experiments CNTL, V1212, V3030, and V3012.

velocity and reflectivity simultaneously: VF3012 and VF3012A. In the following we will compare these three experiments.

\section{1) VERIFICATION OF STATE VARIABLES}

Figure 16 shows the vertical profiles of RMSEs of the analysis against the truth simulation for $U, V, T$, and $Q$. The analysis errors of $U, V$ for the three assimilation experiments are similar, and V3012 has smaller errors of $U$ and $V$ in the middle levels and VF3012A has smaller errors of $V$ below $850 \mathrm{hPa}$. The analysis errors of $T$ for V3012 are least below $500 \mathrm{hPa}$, and VF3012 has smaller errors of $T$ than that of VF3012A below $850 \mathrm{hPa}$. The analysis errors of $Q$ are reduced when the estimated water vapor from reflectivity observations are assimilated, and VF3012 has the least errors with VF3012A second to it. The vertical profiles of RMSEs for the 6-h forecasts are shown in Fig. 17. The forecast errors of $U$ and $V$ for VF3012A are the least among the three experiments in the levels above $600 \mathrm{hPa}$, and VF3012 has the least forecast errors of $U$ and $V$ in the levels below $600 \mathrm{hPa}$. For the variable $T$ below $400 \mathrm{hPa}$,
VF3012 has the least forecast errors, and VF3012A is second to it. For the variable $Q$, VF3012 and VF3012A have similar forecast errors, and they both outperform V3012 due to the assimilation of reflectivity.

\section{2) QuANTITATIVE PRECIPITATION FORECAST}

Figure 18 shows the 6-h accumulated precipitation forecasts of the TRUTH, V3012, VF3012A, and VF3012. With the additional reflectivity assimilated, both of VF3012A and VF3012 improve the precipitation simulation in area B where V3012 only predicts scattered precipitation. VF3012 slightly outperforms VF3012A in area $\mathrm{C}$ where the latter overestimates the precipitation. The results of FSSs of 6-h accumulated precipitation for V3012, VF3012A, and VF3012 are shown in Fig. 19. After the reflectivity is assimilated with MTS4DVar, the precipitation scores of VF3012A and VF3012 are higher than that of V3012 for almost all thresholds, and VF3012 has relatively greater values than VF3012A. The shorter time window employed in the second outer loop in VF3012 can reduce the nonlinearity of radar reflectivity assimilation, and it may explain why there 

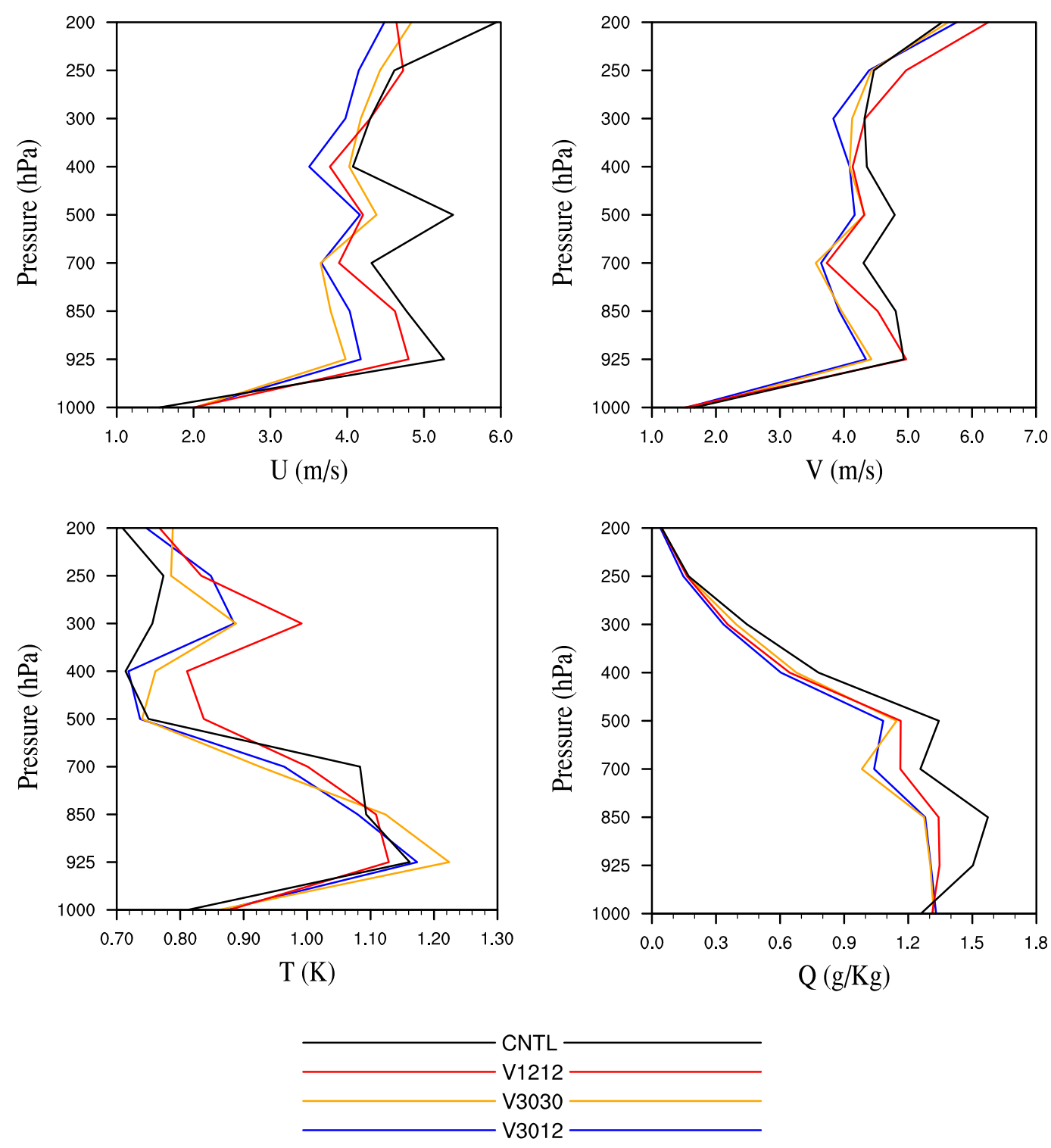

FIG. 15. As in Fig. 10, but for the 6-h forecast.

is no advantage in withholding the reflectivity data in later time levels in the longer window assimilation in MTS-4DVar.

\section{Conclusions}

To assimilate multiscale information from observations at multiple times, an MTS-4DVar scheme was developed. The MTS-4DVar employed multitime windows in the framework of incremental WRFDA 4DVar, with longer windows employed in the first several outer loops to assimilate relatively larger-scale information and shorter windows in the last several outer loops to assimilate more localized features from observations. To evaluate the application of the MTS4DVar on radar data assimilation, a series of single observation tests and OSSEs with multitime windows by assimilating simulated radial velocity and reflectivity were carried out.

The detailed descriptions of the algorithm of MTS4DVar are first discussed. The optimal analysis of 4DVar typically emphasizes the time scales of the atmosphere that is simulated by the forward model within the given time window. The analysis process of MTS-4DVar is a series of optimal solutions from the longer time window to the shorter window. With the first guess from the previous longer-window solution and the improved linearization trajectory in 

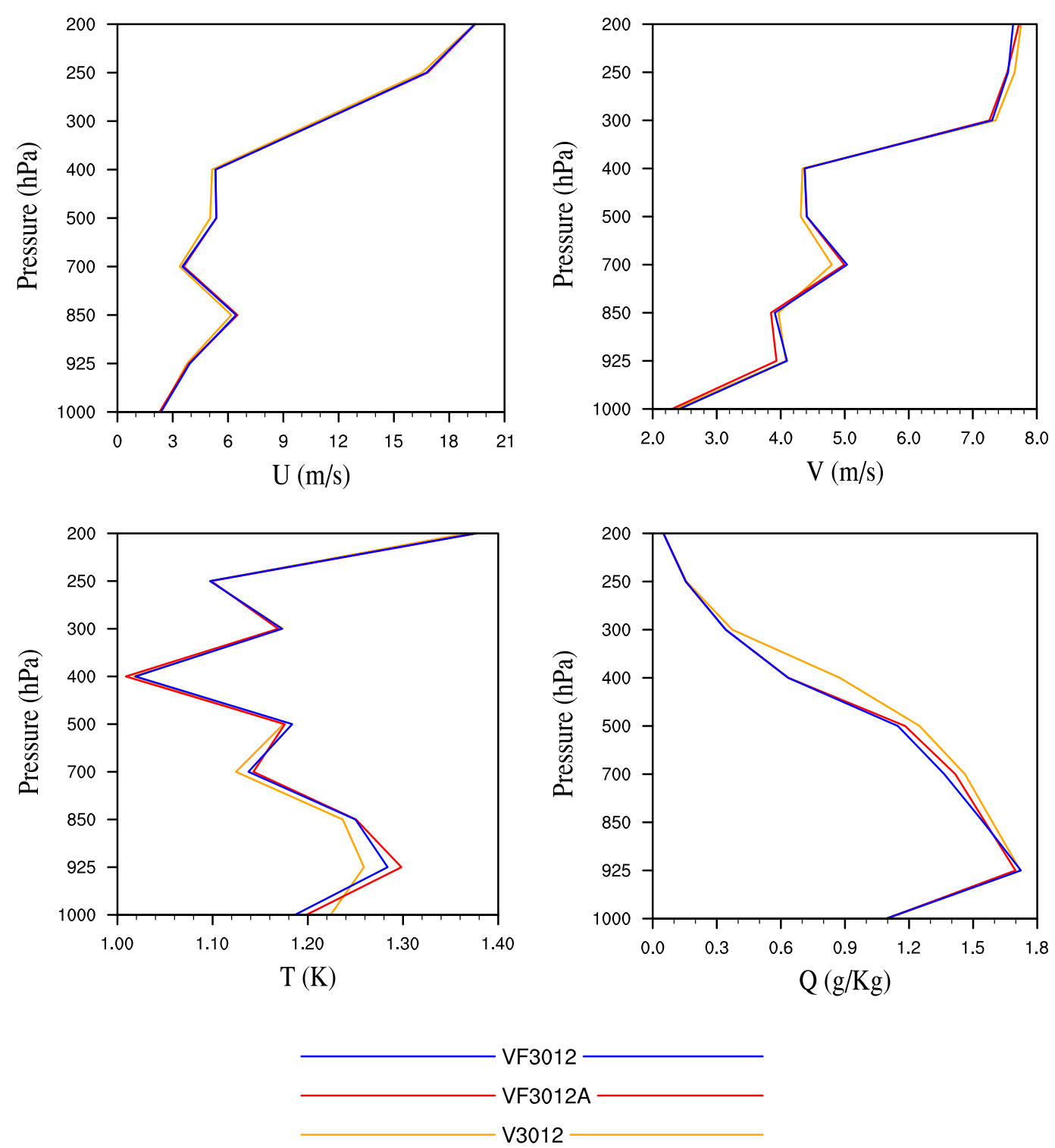

FIG. 16. Vertical profiles of RMSE of the analysis against the truth simulation for (a) $U$, (b) $V$, (c) $T$, and (d) $Q$ of V3012, VF3012A, and VF3012.

the successive shorter-window outer loop, it is anticipated that the multiscale information from observations can be extracted in the optimal analysis of MTS-4DVar. The nonlinearity propagation of variables related to radial velocity and reflectivity were then diagnosed using the NLI and CII. It was shown that the nonlinearity growth of reflectivity is faster than that of radial velocity, indicating that the time window of assimilating reflectivity should be shorter.

Single radial velocity and reflectivity observation assimilation experiments were carried out to show how the wind information was spread spatially and propagated to other variables with multitime windows employed in incremental 4DVar. The results showed that the longer time window in 4DVar produced longer-range increments that might represent larger-scale motions, while the shorter 4DVar window contained more localized features. The MTS-4DVar produced a reasonable combination of the larger-scale information and the localized features in wind increments with multitime windows employed. The longer window employed in 4DVar ensured sufficient time to transfer radial velocity information to temperature and humidity variables, but it may bring negative impacts to the analysis 

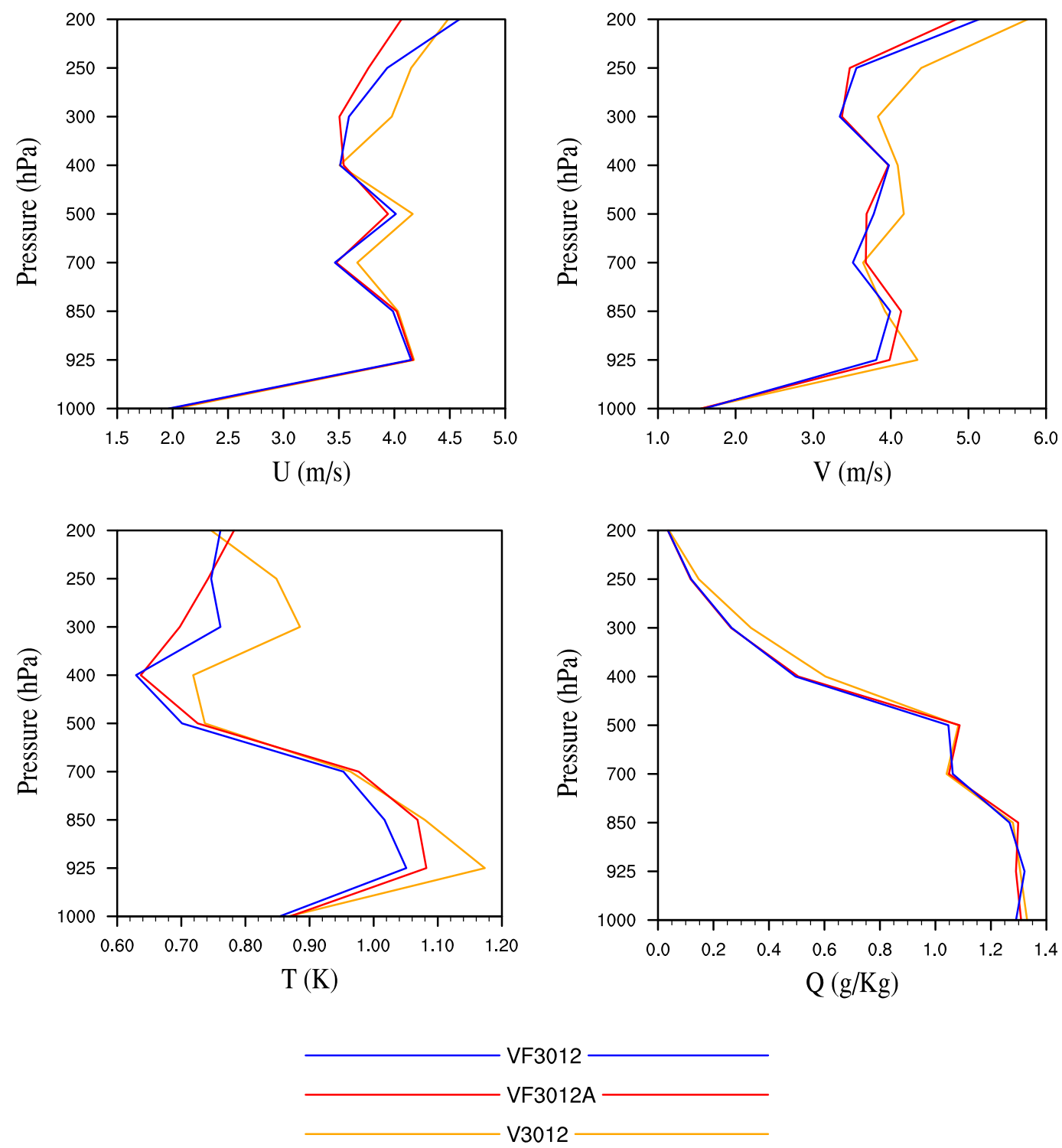

FIG. 17. As in Fig. 14, but for 6-h forecasts.

when the reflectivity observations are assimilated, likely due to the rapid nonlinear growth of hydrometeor variables.

The capability of the MTS-4DVar in radar assimilation were further evaluated by conducting OSSEs. The results of the minimization showed that the cost functions of MTS-4DVar were reduced properly with the iterative updates of the basic states, and the shorter window helped the MTS-4DVar converge well. Benefited from the multitime windows employed in MTS-4DVar, the multiscale dynamic features were well combined in the wind increments. The use of a longer window allowed 4D-Var to attain better multivariate analyses of temperature and humidity fields, and these analyses were well maintained during the shorter window assimilation in the second outer loop in MTS-4DVar. With the improved analysis fields in MTS-4DVar, the intensity and location of the 6-h accumulated precipitation were well improved, and the forecast errors were reduced for the velocity, temperature, and humidity fields. The results of the simultaneous assimilation of radial velocity and reflectivity using MTS-4DVar showed that the added reflectivity observations further improved the humidity analysis and thus the forecasts of model state variables, resulting in improved precipitation forecasts. Results also showed that there are no clear advantages in withholding the reflectivity data in later time levels in the longer 

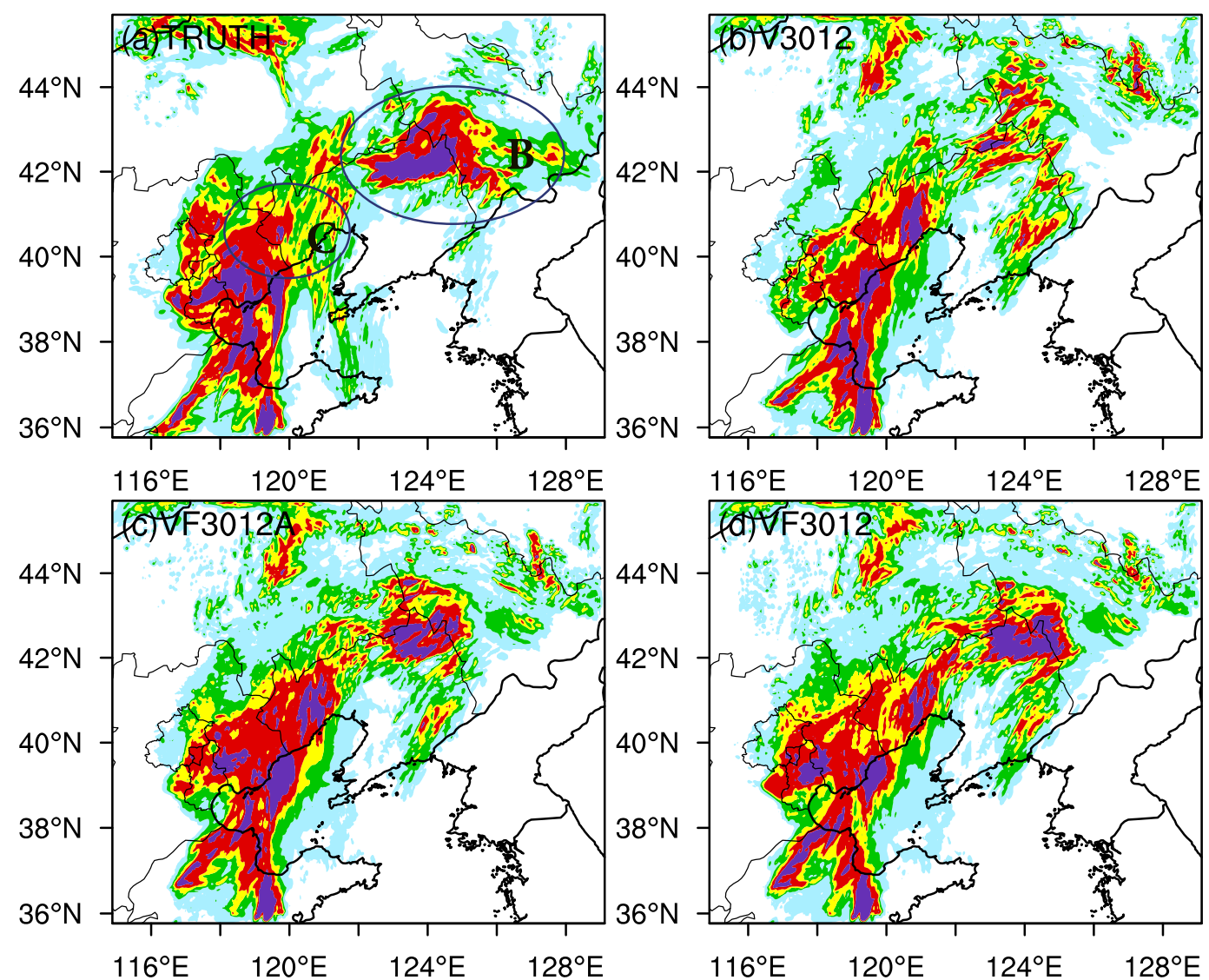

$116^{\circ} \mathrm{E} \quad 120^{\circ} \mathrm{E} \quad 124^{\circ} \mathrm{E} \quad 128^{\circ} \mathrm{E}$

$116^{\circ} \mathrm{E} \quad 120^{\circ} \mathrm{E} \quad 124^{\circ} \mathrm{E} \quad 128^{\circ} \mathrm{E}$

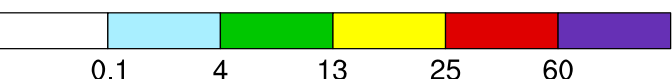

FIG. 18. The 6-h accumulated precipitation (mm) initialized from 1800 UTC 2 Aug 2017 for (a) TRUE, (b) V3012 (c) VF3012A, and (d) VF3012.

window assimilation in MTS-4DVar, meaning that the MTS-4DVar can be well applied to reflectivity assimilation.

Both the single observation assimilation experiments and the OSSEs show that the MTS-4DVar had the capability to assimilate multiscale information from radar radial velocity and reflectivity observations. Due to the limit of computation cost, the MTS-4DVar was only evaluated with OSSEs for a rainfall case, and further study will focus on the applicability of the MTS_4DVar to real data assimilation with cycling configurations. In addition to the assimilation of radial velocity and reflectivity, the MTS-4DVar can also be applied to the assimilation of observations with high temporal and spatial resolution, such as the newgeneration geostationary satellite radiances (Wang et al. 2018), the dual-polarization radar observations (Kawabata et al. 2018). Moreover, while the longer window used in the first outer loop is only $30 \mathrm{~min}$ in the current study in order to effectively extract information from radar observations, a much larger window length can be applied to infer synoptic-scale information from

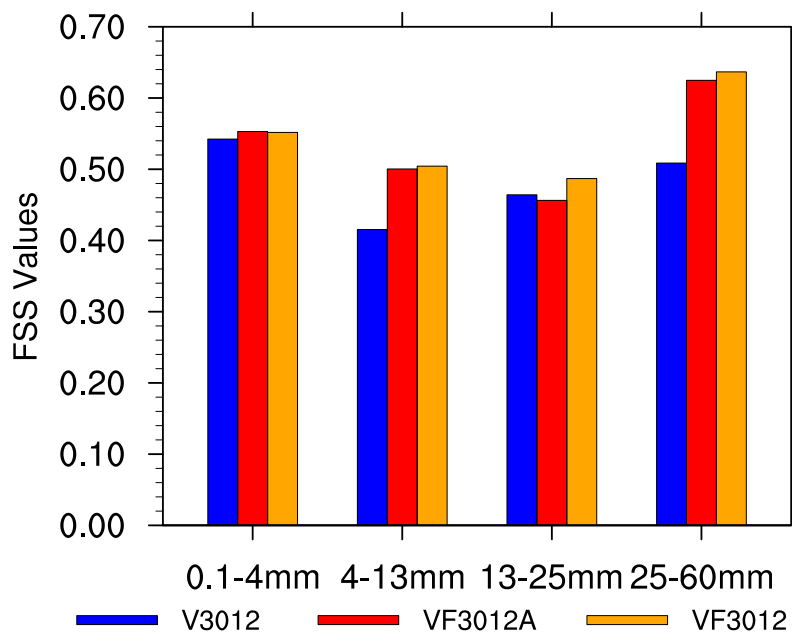

FIG. 19. The FSSs of 6-h accumulated precipitation with different thresholds for V3012, VF3012A, and VF3012. 
conventional observations. These ideas will be explored in future work.

Acknowledgments. This work was jointly sponsored by the National Key Research and Development Program of China (2017YFC1502102) and National Natural Science Foundation of China (41675102).

\section{REFERENCES}

Aranami, K., and Coauthors, 2015: A new operational regional model for convection-permitting numerical weather prediction at JMA. CAS/JSC WGNE Res. Activ. Atmos. Oceanic Model., 45, 0505-0506.

Bonavita, M., P. Lean, and E. Holm, 2018: Nonlinear effects in 4D-Var. Nonlinear Processes Geophys., 25, 713-729, https:// doi.org/10.5194/npg-25-713-2018.

Choi, Y., G. H. Lim, and D. K. Lee, 2013: Radar radial wind data assimilation using the time-incremental 4D-Var method implemented to the WRFDA system. Tellus, 65A, 19677, https:// doi.org/10.3402/tellusa.v65i0.19677.

Courtier, P., J.-N. Thépaut, and A. Hollingsworth, 1994: A strategy for operational implementation of 4D-Var, using an incremental approach. Quart. J. Roy. Meteor. Soc., 120, 1367-1387, https://doi.org/10.1002/qj.49712051912.

Dowell, D. C., L. J. Wicker, and C. Snyder, 2011: Ensemble Kalman filter assimilation of radar observations of the 8 May 2003 Oklahoma City supercell: Influences of reflectivity observations on storm-scale analyses. Mon. Wea. Rev., 139, 272 294, https://doi.org/10.1175/2010MWR3438.1.

Dudhia, J., 1989: Numerical study of convection observed during the winter monsoon experiment using a mesoscale two-dimensional model. J. Atmos. Sci., 46, 3077-3107, https://doi.org/10.1175/ 1520-0469(1989)046<3077:NSOCOD>2.0.CO;2.

Evensen, G., 1994: Sequential data assimilation with a nonlinear quasi-geostrophic model using Monte Carlo methods to forecast error statistics. J. Geophys. Res., 99, 10143-10162, https://doi.org/10.1029/94JC00572.

_ 2004: Sampling strategies and square root analysis schemes for the EnKF. Ocean Dyn., 54, 539-560, https:/doi/org/10.1007/ s10236-004-0099-2.

Fabry, F., 2010: For how long should what data be assimilated for the mesoscale forecasting of convection and why? Part II: On the observation signal from different sensors. Mon. Wea. Rev., 138, 256-264, https://doi.org/10.1175/2009MWR2884.1. , and J. Sun, 2010: For how long should what data be assimilated for the mesoscale forecasting of convection and why? Part I: On the propagation of initial condition errors and their implications for data assimilation. Mon. Wea. Rev., 138, 242255, https://doi.org/10.1175/2009MWR2883.1.

Gao, J., and D. J. Stensrud, 2012: Assimilation of reflectivity data in a convective-scale, cycled 3DVAR framework with hydrometeor classification. J. Atmos. Sci., 69, 1054-1065, https:// doi.org/10.1175/JAS-D-11-0162.1.

$\longrightarrow$, and - 2014: Some observing system simulation experiments with a hybrid 3DEnVAR system for storm-scale radar data assimilation. Mon. Wea. Rev., 142, 3326-3346, https:// doi.org/10.1175/MWR-D-14-00025.1.

Gustafsson, N., and Coauthors, 2018: Survey of data assimilation methods for convective-scale numerical weather prediction at operational centres. Quart. J. Roy. Meteor. Soc., 144, 12181256, https://doi.org/10.1002/qj.3179.
Hong, S., Y. Noh, and J. Dudhia, 2006: A new vertical diffusion package with an explicit treatment of entrainment processes. Mon. Wea. Rev., 134, 2318-2341, https://doi.org/10.1175/MWR3199.1.

Houtekamer, P. L., and H. L. Mitchell, 2005: Ensemble Kalman filtering. Quart. J. Roy. Meteor. Soc., 131, 3269-3289, https:// doi.org/10.1256/qj.05.135.

Huang, X., and Coauthors, 2009: Four-dimensional variational data assimilation for WRF: Formulation and preliminary results. Mon. Wea. Rev., 137, 299-314, https://doi.org/10.1175/ 2008MWR2577.1.

Kawabata, T., T. Kuroda, H. Seko, and K. Saito, 2011: A cloudresolving 4D-Var assimilation experiment for a local heavy rainfall event in the Tokyo metropolitan area. Mon. Wea. Rev., 139, 1911-1931, https://doi.org/10.1175/2011MWR3428.1.

_ K. Ito, and K. Satio, 2014: Recent progress of the NHM4DVAR towards a super-high resolution data assimilation. SOLA, 10, 145-149, https://doi.org/10.2151/SOLA.2014-030.

_, T. Schwitalla, A. Adachi, H.-S. Bauer, V. Wulfmeyer, N. Nagumo, and H. Yamauchi, 2018: Observational operators for dual polarimetric radars in variational data assimilation systems (PolRad VAR v1.0). Geosci. Model Dev., 11, 2493 2501, https://doi.org/10.5194/gmd-11-2493-2018.

Klinker, E., F. Rabier, G. Kelly, and J.-F. Mahfouf, 2000: The ECMWF operational implementation of four-dimensional variational assimilation. III: Experimental results and diagnostics with operational configuration. Quart. J. Roy. Meteor. Soc., 126, 1191-1215, https://doi.org/10.1002/qj.49712656417.

Le Dimet, F., and O. Talagrand, 1986: Variational algorithms for analysis and assimilation of meteorological observations: Theoretical aspects. Tellus, 38A, 97-110, https://doi.org/10.3402/ tellusa.v38i2.11706.

Lewis, J., and J. Derber, 1985: The use of adjoint equations to solve a variational adjustment problem with advective constraints. Tellus, 37A, 309-322, https://doi.org/10.1111/j.1600-0870.1985.tb00430.x.

Li, Z., S. P. Ballard, and D. Simonin, 2018: Comparison of 3D-Var and 4D-Var data assimilation in an NWP-based system for precipitation nowcasting at the Met Office. Quart. J. Roy. Meteor. Soc., 144, 404-413, https://doi.org/10.1002/qj.3211.

Lim, K. S. S., and S. Y. Hong, 2010: Development of an effective double-moment cloud microphysics scheme with prognostic cloud condensation nuclei $(\mathrm{CCN})$ for weather and climate models. Mon. Wea. Rev., 138, 1587-1612, https://oi.org/10.1175/ 2009MWR2968.1.

Liu, C., M. Xue, and R. Kong, 2019: Direct assimilation of radar reflectivity data using 3DVAR: Treatment of hydrometeor background errors and OSSE tests. Mon. Wea. Rev., 147, 1729, https://doi.org/10.1175/MWR-D-18-0033.1.

Lorenc, A. C., 2003: Modelling of error covariances by 4D-Var data assimilation. Quart. J. Roy. Meteor. Soc., 129, 3167-3182, https://doi.org/10.1256/qj.02.131.

- and F. Rawlins, 2005: Why does 4D-Var beat 3D-Var? Quart. J. Roy. Meteor. Soc., 131, 3247-3257, https://doi.org/10.1256/qj.05.85.

Mahfouf, J.-F., and F. Rabier, 2000: The ECMWF operational implementation of four-dimensional variational assimilation. II: Experimental results with improved physics. Quart. J. Roy. Meteor. Soc., 126, 1171-1190, https://doi.org/10.1002/qj.49712656416.

Mlawer, E. J., S. J. Taubman, P. D. Brown, M. J. Iacono, and S. A. Clough, 1997: Radiative transfer for inhomogeneous atmospheres: RRTM, a validated correlated-k model for the longwave. J. Geophys. Res., 102, 16 663-16 682, https://doi.org/ 10.1029/97JD00237.

Parrish, D. F., and J. C. Derber, 1992: The National Meteorological Center's spectral statistical-interpolation analysis system. 
Mon. Wea. Rev., 120, 1747-1763, https://doi.org/10.1175/15200493(1992) $120<1747$ :TNMCSS $>2.0$.CO;2.

$\mathrm{Pu}, \mathrm{Z}$., X. Li, and J. Sun, 2009: Impact of airborne Doppler radar data assimilation on the numerical simulation of intensity changes of Hurricane Dennis near a landfall. J. Atmos. Sci., 66, 3351-3365, https://doi.org/10.1175/2009JAS3121.1.

Rabier, F., H. Järvinen, E. Klinker, J.-F. Mahfouf, and A. Simmons, 2000: The ECMWF operational implementation of fourdimensional variational assimilation. I: Experimental results with simplified physics. Quart. J. Roy. Meteor. Soc., 126, 1143-1170, https://doi.org/10.1002/qj.49712656415.

Roberts, N. M., and H. W. Lean, 2008: Scale-selective verification of rainfall accumulations from high-resolution forecasts of convective events. Mon. Wea. Rev., 136, 78-97, https://doi.org/ 10.1175/2007MWR2123.1.

Shen, F., M. Xue, and J. Min, 2017: A comparison of limited-area 3DVAR and ETKF-En3DVAR data assimilation using radar observations at convective scale for the prediction of Typhoon Saomai (2006). Meteor. Appl., 24, 628-641, https://doi.org/ 10.1002/met.1663.

Shen, Y., P. Zhao, Y. Pan, and J. Yu, 2014: A high spatiotemporal gauge-satellite merged precipitation analysis over China. J. Geophys. Res. Atmos., 119, 3063-3075, https://doi.org/10.1002/ 2013JD020686.

Skamarock, W. C., and Coauthors, 2008: A description of the Advanced Research WRF version 3. NCAR Tech. Note NCAR/TN475+STR, 113 pp., https://doi.org/10.5065/D68S4MVH.

Snyder, C., and F. Zhang, 2003: Assimilation of simulated Doppler radar observations with an ensemble Kalman filter. Mon. Wea. Rev., 131, 1663-1677, https://doi.org/10.1175//2555.1.

Sun, J., and N. A. Crook, 1997: Dynamical and microphysical retrieval from Doppler radar observations using a cloud model and its adjoint. Part I: Model development and simulated data experiments. J. Atmos. Sci., 54, 1642-1661, https://doi.org/ 10.1175/1520-0469(1997)054<1642:DAMRFD>2.0.CO;2.

_ _ and H. Wang, 2013: Radar data assimilation with WRF 4DVar. Part II: Comparison with 3D-Var for a squall line over the U.S. Great Plains. Mon. Wea. Rev., 141, 2245-2264, https:// doi.org/10.1175/MWR-D-12-00169.1.

- M. Chen, and Y. Wang, 2010: A frequent-updating analysis system based on radar, surface, and mesoscale model data for the Beijing 2008 forecast demonstration project. Wea. Forecasting, 25, 1715-1735, https://doi.org/10.1175/2010WAF2222336.1.

- , and Coauthors, 2014: Use of NWP for nowcasting convective precipitation: Recent progress and challenges. Bull. Amer. Meteor. Soc., 95, 409-426, https://doi.org/10.1175/BAMS-D11-00263.1.

, H. Wang, W. Tong, Y. Zhang, C. Lin, and D. Xu, 2016: Comparison of the impacts of momentum control variables on high-resolution variational data assimilation and precipitation forecasting. Mon. Wea. Rev., 144, 149-169, https://doi.org/ 10.1175/MWR-D-14-00205.1.
Tiedtke, M., 1989: A comprehensive mass flux scheme for cumulus parameterization in large-scale models. Mon. Wea. Rev., 117, 1779-1800, https://doi.org/10.1175/1520-0493(1989)117<1779: ACMFSF $>2.0 . \mathrm{CO} ; 2$

Tong, M., and M. Xue, 2005: Ensemble Kalman filter assimilation of Doppler radar data with a compressible nonhydrostatic model: OSS experiments. Mon. Wea. Rev., 133, 1789-1807, https://doi.org/10.1175/MWR2898.1.

Trémolet, Y., 2006: Accounting for an imperfect model in 4D-Var. Quart. J. Roy. Meteor. Soc., 132, 2483-2504, https://doi.org/ 10.1256/qj.05.224.

Wang, H., T. Auligné, and H. Morrison, 2012: Impact of microphysics scheme complexity on the propagation of initial perturbations. Mon. Wea. Rev., 140, 2287-2296, https://doi.org/ 10.1175/MWR-D-12-00005.1.

_ J. Sun, S. Fan, and X. Huang, 2013a: Indirect assimilation of radar reflectivity with WRF $3 \mathrm{D}$-Var and its impact on prediction of four summertime convective events. J. Appl. Meteor. Climatol., 52, 889-902, https://doi.org/10.1175/JAMC-D-12-0120.1.

,$- \ldots$, X. Zhang, X. Huang, and T. Auligné, 2013b: Radar data assimilation with WRF 4D-Var. Part I: System development and preliminary testing. Mon. Wea. Rev., 141, 2224-2244, https://doi.org/10.1175/MWR-D-12-00168.1.

Wang, Y., Z. Liu, S. Yang, J. Min, L. Chen, Y. Chen, and T. Zhang, 2018: Added value of assimilating Himawari-8 AHI water vapor radiances on analyses and forecasts for "7.19" severe storm over north China. J. Geophys. Res. Atmos., 123, 33743394, https://doi.org/10.1002/2017JD027697.

Xiao, Q., Y. Kuo, J. Sun, W. Lee, E. Lim, Y. Guo, and D. M. Barker, 2005: Assimilation of Doppler radar observations with a regional 3DVAR system: Impact of Doppler velocities on forecasts of a heavy rainfall case. J. Appl. Meteor., 44, 768788, https://doi.org/10.1175/JAM2248.1.

,,,,--- D. M. Barker, and E. Lim, 2007: An approach of radar reflectivity data assimilation and its assessment with the inland QPF of Typhoon Rusa (2002) at landfall. J. Appl. Meteor. Climatol., 46, 14-22, https://doi.org/10.1175/JAM2439.1.

Xue, M., M. Tong, and K. K. Droegemeier, 2006: An OSSE framework based on the ensemble square root Kalman filter for evaluating the impact of data from radar networks on thunderstorm analysis and forecasting. J. Atmos. Oceanic Technol., 23, 46-66, https://doi.org/10.1175/JTECH1835.1.

Zhang, B., X. Tian, J. Sun, F. Chen, L. Zhang, and S. Fu, 2015: PODEn4DVar-based radar data assimilation scheme: Formulation and preliminary results from real-data experiments with advanced research WRF (ARW). Tellus, 67A, 26045, https:// doi.org/10.3402/tellusa.v67.26045.

Zhang, X., X. Huang, J. Liu, J. Poterjoy, Y. Weng, F. Zhang, and H. Wang, 2014: Development of an efficient regional fourdimensional variational data assimilation system for WRF. J. Atmos. Oceanic Technol., 31, 2777-2794, https://doi.org/ 10.1175/JTECH-D-13-00076.1. 